FIAN-TD-2014-02

\title{
Higher-Spin Theory and Space-Time Metamorphoses
}

\author{
M.A. Vasiliev \\ I.E. Tamm Department of Theoretical Physics, Lebedev Physical Institute, \\ Leninsky prospect 53, 119991, Moscow, Russia
}

\begin{abstract}
Introductory lectures on higher-spin gauge theory given at 7 Aegean workshop on non-Einstein theories of gravity. The emphasis is on qualitative features of the higherspin gauge theory and peculiarities of its space-time interpretation. In particular, it is explained that Riemannian geometry cannot play a fundamental role in the higher-spin gauge theory. The higher-spin symmetries are argued to occur at ultra high energy scales beyond the Planck scale. This suggests that the higher-spin gauge theory can help to understand Quantum Gravity. Various types of higher-spin dualities are briefly discussed.
\end{abstract}




\section{Contents}

1 Introduction

2 Lower-spin global symmetries

3 Local Symmetries 5

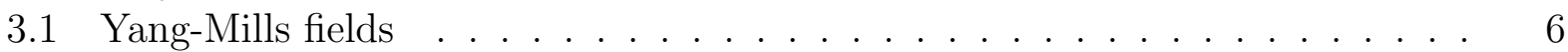

3.2 Einstein-Cartan gravity and supergravity . . . . . . . . . . . 6

3.3 Spontaneous symmetry breaking . . . . . . . . . . . . . 7

4 General Properties of HS Theory

4.1 Fronsdal fields . . . . . . . . . . . . . . . . . . . . 7

4.2 No-go and the role of $(A) d S \ldots \ldots \ldots$

4.3 HS Symmetries versus Riemannian geometry . . . . . . . . . . . . 8

4.4 HS gauge theory, Quantum Gravity and String Theory . . . . . . . . . . 9

4.5 Higher-spin AdS/CFT correspondence . . . . . . . . . . . . . . . 9

5 Global HS Symmetry: Idea of Construction 10

5.1 Auxiliary problem ..................... . . . . . . . . . . . . . . . .

5.2 Massless scalar field unfolded . . . . . . . . . . . . . . .

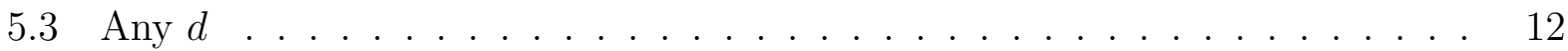

6 Conformal HS Algebra in $d=3$

$6.13 d$ multispinors . . . . . . . . . . . . . . . . . . 12

6.2 Spinorial form of $3 d$ massless equations . . . . . . . . . . . . . . 13

$6.33 d$ HS symmetry . . . . . . . . . . . . . . . . . . . 13

6.4 Weyl algebra and star product . . . . . . . . . . . . . . 14

7 HS Symmetry in $A d S_{4}$

7.1 Spinor language in four dimension . . . . . . . . . . . . . . . . 15

$7.2 A d S_{4} \mathrm{HS}$ algebra ........................ . . . . . . . . .

8 Free HS fields in four dimension

8.1 Vacuum solution ...................... . . . . . . . . . . . . .

8.1.1 Central on-shell theorem . . . . . . . . . . . . . . . . 17

8.1 .2 Examples ......................... 18

9 Nonlinear higher-spin theory $\quad \mathbf{1 9}$

9.1 Idea of construction . . . . . . . . . . . . . . . . . . . . . 19

9.2 HS star product . . . . . . . . . . . . . . . . . . . . . . . . . . . . . . . . . . . . .

9.3 The full nonlinear system . . . . . . . . . . . . . . . . . . . . . . . . . . . . . . .

9.4 Properties of HS interactions . . . . . . . . . . . . . . . 23 
10 Unfolded Dynamics 24

10.1 General setup . . . . . . . . . . . . . . . . . . 24

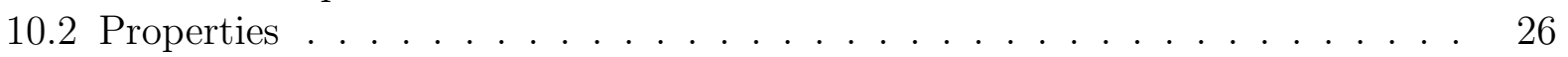

11 Space-time metamorphoses 26

$11.1 A d S_{4} / C F T_{3}$ HS holography $\ldots \ldots \ldots \ldots \ldots \ldots \ldots$

$11.2 s p(8)$ invariant setup . . . . . . . . . . . . . . . . 28

11.2.1 From four to ten . . . . . . . . . . . . . . . 29

11.2 .2 From ten to four . . . . . . . . . . . . . . . . . 30

12 HS theory and quantum mechanics 31

13 To String Theory via Multiparticle Symmetry 32

14 Summary and Conclusion 32 


\section{Introduction}

Higher-spin (HS) gauge theories form a class of theories exhibiting infinite-dimensional symmetries which go beyond conventional lower-spin symmetries. The primary goal of these lectures is to focus on qualitative aspects of HS gauge theories avoiding technical details as much as possible. The emphasis is on possible consequences of HS symmetries for our understanding of space-time. It will be explained in particular that in the setup of HS gauge theories the usual concepts of Riemannian geometry such as metric, local event and spacetime dimension cannot play a fundamental role. The HS symmetries will be argued to occur at ultra high energy scales beyond the Planck scale. Having a potential to describe transPlanckian energies, HS gauge theory can shed light on the problem of Quantum Gravity. Various aspects of HS dualities including $A d S / C F T$ and duality with quantum mechanics are briefly discussed.

\section{Lower-spin global symmetries}

The fundamental example of a lower-spin symmetry is provided by the Poincaré symmetry which underlies relativistic theories. It acts on coordinates of Minkowski space-time as $\delta x^{a}=\epsilon^{a}+\epsilon_{b}^{a} x^{b}$ where $\epsilon^{a}$ and $\epsilon^{a b}$ are parameters of infinitesimal translations and Lorentz rotations, respectively. One can write

$$
\delta x^{a}=\left[T, x^{a}\right], \quad T=\epsilon^{n} P_{a}+\epsilon^{a b} M_{a b},
$$

where

$$
P_{a}=\frac{\partial}{\partial x^{a}}, \quad M_{a b}=x_{a} \frac{\partial}{\partial x^{b}}-x_{b} \frac{\partial}{\partial x^{a}}
$$

are the generators of the Poincaré algebra $i s o(d-1,1)$ obeying the commutation relations

$$
\begin{gathered}
{\left[M_{a b}, P_{c}\right]=P_{a} \eta_{b c}-P_{b} \eta_{a c},} \\
{\left[M_{a b}, M_{c d}\right]=M_{a d} \eta_{b c}-M_{b d} \eta_{a c}-M_{a c} \eta_{b d}+M_{b c} \eta_{a d},} \\
{\left[P_{a}, P_{b}\right]=0}
\end{gathered}
$$

where $\eta_{a b}$ is the Minkowski metric.

The Poincaré algebra admits the (anti-) de Sitter deformation $l$ with

$$
\left[P_{a}, P_{b}\right]=\Lambda M_{a b}
$$

which describes symmetries of either anti-de Sitter space at $\Lambda<0(l=o(d-1,2))$ or de Sitter space at $\Lambda>0(l=o(d, 1))$. At $\Lambda=0, l=i s o(d-1,1)$ describes the symmetries of Minkowski space.

Supersymmetry is the extension of the Poincaré symmetry by supergenerators $Q_{A}$ obeying relations

$$
\left\{Q_{A}, Q_{B}\right\}=\gamma_{A B}^{a} P_{a},
$$




$$
\left[M_{a b}, Q_{A}\right]=\sigma_{a b A}^{B} Q_{B}, \quad \sigma_{a b}=\frac{1}{4}\left[\gamma_{a}, \gamma_{b}\right],
$$

where $A, B=1,2,3,4$ are the Majorana spinor indices in four dimensions. Note that, being fermions, supergenerators obey anticommutation relations.

Internal symmetry generators $T_{i}$ are space-time invariant

$$
\left[T_{i}, P_{a}\right]=0, \quad\left[T^{i}, M_{a b}\right]=0 .
$$

In particular, the symmetries of the Standard Model $T_{i} \in s u(3) \times s u(2) \times u(1)$ are of this type.

To complete the list of symmetries that play a role in conventional lower-spin theories it remains to mention conformal (super)symmetries. These will be discussed in some more detail below.

\section{Local Symmetries}

A useful viewpoint is that any global symmetry is the remnant of a local symmetry with parameters like $\varepsilon^{a}(x), \varepsilon^{a b}(x), \varepsilon^{\alpha}(x), \varepsilon^{i}(x)$ being arbitrary functions of space-time coordinates. Local symmetries are symmetries of the full theory. Global symmetries are symmetries of some its particular solution.

For example, the infinitesimal diffeomorphisms $\delta x^{a}=\varepsilon^{a}(x)$ are symmetries of GR while the global symmetries with $\varepsilon^{a}(x)=\epsilon^{a}+\epsilon_{b}^{a} x^{b}$ are symmetries of the Minkowski solution $g_{a b}=\eta_{a b}$ of the Einstein equations.

Let

$$
S=\int_{M^{d}} L\left(\varphi(x), \partial_{a} \varphi(x), \ldots\right)
$$

be invariant under a global symmetry $g$ with parameters $\epsilon^{n}(n=a, \alpha, i, \ldots)$. Letting the symmetry parameters be arbitrary functions of space-time coordinates, $\epsilon^{n} \rightarrow \varepsilon^{n}(x)$, we obtain that

$$
\delta S=-\int_{M^{d}} J_{n}^{a}(\varphi) \partial_{a} \varepsilon^{n}(x)
$$

since $\delta S$ should be zero at $\partial_{a} \varepsilon^{n}(x)=0 . J_{n}^{a}(\varphi)$ are conserved currents since $\partial_{a} J_{n}^{a}(\varphi)=0$ by virtue of the field equations $\delta S=0$.

The local symmetry is achieved with the aid of gauge fields $A_{a}^{n}$ that have the transformation law

$$
\delta A_{a}^{n}=\partial_{a} \varepsilon^{n}+\ldots,
$$

where the ellipsis denotes possible field-dependent terms. The following modification of the action

$$
S \longrightarrow S+\Delta S+\ldots, \quad \Delta S=\int_{M^{d}} J_{n}^{a}(\varphi) A_{a}^{n}(x)
$$

preserves local symmetry in the lowest order in interactions. The term $\Delta S$ describes the so-called Noether current interactions. 
There is, however, a subtlety if $\varphi(x)$ were themselves gauge fields with gauge parameters $\varepsilon^{\prime}$. In this case it may happen that $J_{n}^{a}(\varphi)$ is not invariant under the $\varepsilon^{\prime}$ symmetry. Hence the Noether current interaction for several gauge fields may be obstructed by gauge symmetries.

Localization of various types of lower-spin symmetries leads to important classes of gauge field theories.

\subsection{Yang-Mills fields}

The Yang-Mills theory is responsible for the localization of internal symmetries. For a Lie algebra $l$ with generators $T_{i}$, Yang-Mills fields $A_{a}^{i}(x)$ and symmetry parameters $\varepsilon^{i}$ are valued in $l$

$$
A_{a}(x)=A_{a}^{i}(x) T_{i}, \quad \varepsilon(x)=\varepsilon^{i}(x) T_{i} .
$$

The Yang-Mills gauge transformation is

$$
\delta A_{a}(x)=D_{a} \varepsilon(x)
$$

where

$$
D_{a} \varepsilon(x)=\partial_{a} \varepsilon(x)+\left[A_{a}(x), \varepsilon(x)\right]
$$

is the covariant derivative. The commutator of the covariant derivatives gives the Yang-Mills curvature

$$
\left[D_{a}, D_{b}\right]=R_{a b}, \quad R_{a b}=\partial_{a} A_{b}-\partial_{b} A_{a}+\left[A_{a}, A_{b}\right]
$$

which has the transformation law

$$
\delta R_{a b}=\left[R_{a b}, \varepsilon\right] .
$$

Needless to say that the Yang-Mills fields play a prominent role in the modern theory of non-gravitational fundamental interactions, i.e., the Standard Model.

\subsection{Einstein-Cartan gravity and supergravity}

Localization of the Poincaré symmetry leads to the Cartan formulation of Einstein gravity. The Yang-Mills gauge fields $A_{\nu}^{n}=\left(e_{\nu}{ }^{a}, \omega_{\nu}{ }^{a b}\right)$ associated with the Poincaré algebra include the frame field (vielbein) $e_{\nu}{ }^{a}$ and the Lorentz connection $\omega_{\nu}{ }^{a b}$. The frame field $e_{\nu}{ }^{a}$ relates base indices $\nu$ with the fiber ones $a$. (In Minkowski space in Cartesian coordinates, where $e_{\nu}{ }^{a}=\delta_{\nu}^{a}$, the two types of indices can be identified.) The gauge transformations have the form

$$
\begin{gathered}
\delta e_{\nu}{ }^{a}(x)=\partial_{\nu} \varepsilon^{a}(x)+\omega_{\nu}{ }_{b}{ }_{b}(x) \varepsilon^{b}(x)-\varepsilon^{a}{ }_{b}(x) e_{\nu}{ }^{b}(x)+\Delta e_{\nu}{ }^{a}, \\
\delta \omega_{\nu}{ }^{a b}(x)=\partial_{\nu} \varepsilon^{a b}(x)+\omega_{\nu}{ }^{a}{ }_{c}(x) \varepsilon^{c b}(x)-\omega_{\nu}{ }^{b}{ }_{c}(x) \varepsilon^{c a}(x)+\Delta \omega_{\nu}{ }^{a b} .
\end{gathered}
$$

Here $\Delta e_{\nu}{ }^{a}$ and $\Delta \omega_{\nu}{ }^{a b}$ denote some corrections to the Yang-Mills transformation law, which are proportional to the curvatures

$$
R_{\nu \mu}^{a}=\partial_{\nu} e_{\mu}^{a}+\omega_{\nu}{ }^{a}{ }_{b} e_{\mu}{ }^{b}-(\nu \leftrightarrow \mu), \quad R_{\nu \mu}^{a b}=\partial_{\nu} \omega_{\mu}^{a b}+\omega_{\nu}{ }^{a}{ }_{c} \omega_{\mu}{ }^{c b}-(\nu \leftrightarrow \mu) .
$$


The zero-torsion constraint $R_{\nu \mu}{ }^{a}=0$ expresses the Lorentz connection in terms of the frame field and its derivatives: $\omega=\omega(e, \partial e)$. In this case $R_{\nu \mu}{ }^{\rho \sigma}$ equals to the Riemann tensor. Recall that the relation of the metric with the frame field is $g_{\nu \mu}=e_{\nu}{ }^{a} e_{\mu}{ }^{b} \eta_{a b}$.

Localization of supersymmetry extends the gravitational fields by the spin- $3 / 2$ gauge field gravitino $\psi_{\nu \alpha}$ with the gauge transformation law

$$
\delta \psi_{\nu \alpha}=D_{\nu} \varepsilon_{\alpha}+\ldots .
$$

Gauge theories of this type are called supergravities, constituting a very interesting class of extensions of the theory of gravity. (See e.g. [1] and references therein. Note that the construction of supergravity in terms of the gauge fields of the supersymmetry algebra was suggested in [2].)

\subsection{Spontaneous symmetry breaking}

Generally, one should distinguish between the symmetry $G$ of some equations and a symmetry $\tilde{G}$ of some their particular solution. For example, for the case of the Higgs field $H^{i}(x)=$ $H_{0}^{i}+h^{i}(x)$, the unbroken part $\tilde{G} \subset G$ is a residual symmetry of $H_{0}^{i}: \tilde{G}=S U(3) \times U(1)$ in the Standard Model. For $H_{0}^{i}$ having a non-zero dimension $\left[H_{0}^{i}\right]=\mathrm{cm}^{-1} \sim \mathrm{GeV}$, spontaneous symmetry breaking is a low-energy effect. In other words, the symmetry restores at $E>H_{0}^{i}$.

In the unbroken regime, the gauge fields associated with the usual lower-spin symmetries describe massless particles of spin one $A_{\nu}{ }^{i}$, spin $3 / 2 \psi_{\nu \alpha}$ and spin two $e_{\nu}{ }^{a}, \omega_{\nu}{ }^{a b}$.

\section{General Properties of HS Theory}

The key question is whether it is possible to go to larger HS symmetries. If yes, what are HS symmetries and HS counterparts of the lower-spin theories including GR? What are physical motivations for their study and possible outputs?

\subsection{Fronsdal fields}

As shown by Fronsdal [3], all symmetric massless HS fields are gauge fields. They are described by rank- $s$ symmetric tensors $\phi_{\nu_{1} \ldots \nu_{s}}$ obeying the double tracelessness condition $\phi^{\rho}{ }_{\rho}^{\mu}{ }_{\mu \nu_{5} \ldots \nu_{s}}=0$. The gauge transformation is

$$
\delta \phi_{\nu_{1} \ldots \nu_{s}}(x)=\partial_{\left(\nu_{1}\right.} \varepsilon_{\left.\nu_{2} \ldots \nu_{s}\right)}(x),
$$

where the gauge parameter is symmetric and traceless

$$
\varepsilon^{\mu}{ }_{\mu \nu_{3} \ldots \nu_{s-1}}=0 .
$$

The field equations have the form

$$
\mathcal{R}_{\nu_{1} \ldots \nu_{s}}(x)=0,
$$


where the Ricci-like tensor $\mathcal{R}_{\nu_{1} \ldots \nu_{s}}(x)$ is

$$
\mathcal{R}_{\nu_{1} \ldots \nu_{s}}(x)=\square \phi_{\nu_{1} \ldots \nu_{s}}(x)-s \partial_{\left(\nu_{1}\right.} \partial^{\mu} \phi_{\left.\nu_{2} \ldots \nu_{s} \mu\right)}(x)+\frac{s(s-1)}{2} \partial_{\left(\nu_{1}\right.} \partial_{\nu_{2}} \phi_{\left.\nu_{3} \ldots \nu_{s} \mu\right)}^{\mu}(x) .
$$

The gauge invariant Fronsdal action is

$$
S=\int_{M^{d}}\left(\frac{1}{2} \phi^{\nu_{1} \ldots \nu_{s}} \mathcal{R}_{\nu_{1} \ldots \nu_{s}}(\phi)-\frac{1}{8} s(s-1) \phi_{\mu}{ }^{\mu \nu_{3} \ldots \nu_{s}} \mathcal{R}_{\rho \nu_{3} \ldots \nu_{s}}^{\rho}(\phi)\right)
$$

\subsection{No-go and the role of $(A) d S$}

In the sixties of the last century it was argued by Weinberg [4] and Coleman and Mandula [5] that HS symmetries cannot be realized in a nontrivial local field theory in Minkowski space. In the seventies it was shown by Aragone and Deser [6] that HS gauge symmetries are incompatible with GR within an expansion over Minkowski space. The general belief was that nontrivial interactions of massless HS fields cannot be introduced.

Nevertheless, in the eighties, it was shown by light-cone [7, 8] and covariant methods [9, [10] that some non-gravitational HS interactions can be constructed at least at the cubic order. These results suggested that some consistent HS theory should exist.

The further progress resulted from the observation that the consistent formulation of the HS gauge theory requires a curved background instead of the flat Minkowski. The most symmetric curved cousins of the flat Minkowski space are de Sitter and anti-de Sitter spaces. That HS theories admit consistent interactions including the gravitational interaction in (A) $d S$ background was shown in [11, 12]. In agreement with the no-go statements, the limit of zero cosmological constant $\Lambda \rightarrow 0$ turns out to be singular so that, indeed, HS theories with unbroken HS symmetries do not exist in the Minkowski background.

\subsection{HS Symmetries versus Riemannian geometry}

The HS symmetries and the space-time symmetries do not commute simply because HS generators are higher-rank Lorentz tensors

$$
\left[T^{a}, T^{H S}\right]=T^{H S}, \quad\left[T^{a b}, T^{H S}\right]=T^{H S} .
$$

However, the same commutation relations imply that HS generators transform the spacetime generators to the HS generators. Since the gauge fields for space-time generators are the gravitational frame field and Lorentz connection, this implies that HS transformations map the gravitational fields (metric) to the HS fields.

This simple observation has the far-going consequence that the Riemannian geometry is not appropriate for the HS theory, implying in particular that the concept of local event may become illusive in the HS theory!

Though it is not appropriate to use the metric tensor in the HS theory, we do not want to give up the coordinate independence of GR. Fortunately, this can be achieved in the framework of the formalism of differential forms. 
Differential forms are totally antisymmetric tensors. A $p$-form is a rank- $p$ totally antisymmetric tensor $\omega(x)=\theta^{\nu_{1}} \ldots \theta^{\nu_{p}} \omega_{\nu_{1} \ldots \nu_{p}}(x)$ where $\theta^{\nu}$ are anticommuting symbols (variables)

$$
\theta^{\nu} \theta^{\mu}=-\theta^{\mu} \theta^{\nu}
$$

usually called differentials $\theta^{\nu}=d x^{\nu}$. The invariant differentiation is provided by the exterior (de Rham) derivative

$$
d=\theta^{\nu} \frac{\partial}{\partial x^{\nu}}, \quad d^{2}=0 .
$$

This formalism is covariant because, due to the total antisymmetrization of indices, symmetric Christoffel symbols drop out from the covariant derivatives. In this language, the connections $A=\theta^{\nu} A_{\nu}^{i} T_{i}$ are one-forms, while the curvatures $R=D^{2}$ with $D=d+A$ are two-forms.

Farther elaboration of this language in application to HS theory leads eventually to a deeper understanding of fundamental concepts of space-time including its dimension.

\subsection{HS gauge theory, Quantum Gravity and String Theory}

As explained in more detail below, the HS symmetry is in a certain sense maximal relativistic symmetry. Hence one can speculate that it cannot result from spontaneous breakdown of a larger symmetry. This implies that the HS symmetries are manifest at ultrahigh energies above any scale including the Planck scale. If this is true, the HS gauge theory should capture effects of Quantum Gravity. This opens a unique possibility for the theoretical study of the unreachable by experimental tests energy scale of Quantum Gravity by means of the highly restrictive HS symmetry.

On the other hand, since the lower-spin symmetries are subalgebras of the HS symmetries, it is natural to expect that the lower-spin theories can correspond to low-energy limits of the HS theory with spontaneously broken HS symmetries.

A related issue is a connection of HS theory with String Theory. A natural conjecture is that String Theory can be interpreted as a spontaneously broken theory of the HS type, where $s>2$ fields acquire nonzero masses. An interesting recent conjecture [13] is that String Theory can be identified with the full quantum HS theory.

\subsection{Higher-spin $A d S / C F T$ correspondence}

That the HS gauge theories are most naturally formulated in the anti-de Sitter background makes them interesting from the perspective of $A d S / C F T$ correspondence [14, 15, 16]. Various aspects of the HS holography were discussed by many authors starting from [17, 18, 19] (see also [20, 21]). However, the concrete proposal is due to Klebanov and Polyakov [22] who conjectured that the $A d S_{4}$ HS theory is dual to $3 d$ the vectorial conformal models. This hypothesis was successfully checked by Giombi and Yin [23, that triggered a lot of interest to the HS holography. The conjecture of Klebanov and Polyakov was later extended to the fermionic boundary systems [24, 25] as well to the $A d S_{3} / C F T_{2}$ correspondence [26, 27, 28]. 
The HS holography has several features which give a hope that its analysis may help to uncover the origin of $A d S / C F T$. Indeed, as discussed in some more detail below, a progress in this direction has been achieved in [29]. It should be stressed that the HS holography does not rely on supersymmetry and is a weak-weak duality that therefore can be checked directly on the both sides. For more detail on the HS holography we refer the reader to 30, 31.

\section{Global HS Symmetry: Idea of Construction}

The simplest way to figure out what is a HS symmetry is via the $A d S / C F T$ correspondence. Namely, the global HS symmetry of the most symmetric $A d S_{d+1}$ solution can be identified with the maximal symmetry of the $d$-dimensional free conformal fields. In the most cases the latter are identified with the massless scalar and/or spinor.

Consider KG massless equation in $d$-dimensional Minkowski space

$$
\square C(x)=0, \quad \square=\eta^{a b} \frac{\partial^{2}}{\partial x^{a} \partial x^{b}} .
$$

The conformal HS symmetry is the symmetry of this equation. What is this symmetry? Its structure was first elaborated for $d=3$ in [32] and soon after by Eastwood [33] for any $d$.

Of course, this symmetry contains the Poincaré transformations as well as the scale transformation (dilatation)

$$
\delta C(x)=\varepsilon D C(x), \quad D=x^{a} \frac{\partial}{\partial x^{a}}+\frac{d}{2}-1
$$

and the special conformal transformations

$$
\delta C(x)=\varepsilon_{a} K^{a} C(x), \quad K^{a}=\left(x^{2} \eta^{a b}-2 x^{a} x^{b}\right) \frac{\partial}{\partial x^{b}}+(2-d) x^{a} .
$$

Altogether $P_{a}, M_{a b}, K^{a}$ and $D$ form the conformal Lie algebra $o(d, 2)$.

To figure out the structure of the whole conformal HS algebra it is useful to consider an auxiliary problem.

\subsection{Auxiliary problem}

Consider the equations

$$
D \mathcal{C}_{A}(x)=0,
$$

where $\mathcal{C}_{A}(x)$ is a set of fields valued in some space $V$ (the label $A$ ) and

$$
D=d+\omega(x), \quad \omega_{A}^{B}(x)=\omega^{\Omega}(x) T_{\Omega A}^{B}
$$

is a covariant derivative acting in the space $V$ treated as a $g l(V)$-module. i.e., $\omega(x)$ is some $g l(V)$-connection. The covariant derivative $D$ is demanded to be flat, i.e.,

$$
D^{2}=0 \text {. }
$$


Clearly, Eqs. (5.1) and (5.2) are invariant under the gauge transformation

$$
\begin{gathered}
\delta \mathcal{C}_{A}(x)=-\varepsilon_{A}{ }^{B}(x) \mathcal{C}_{B}(x), \quad \varepsilon_{A}{ }^{B}(x)=\varepsilon^{\Omega}(x) T_{\Omega A}{ }^{B}(x), \\
\delta \omega(x)=D \varepsilon(x):=d \varepsilon(x)+\omega(x) \varepsilon(x)-\varepsilon(x) \omega(x),
\end{gathered}
$$

where indices are implicit. The condition that the equations remain invariant for some fixed $\omega(x)=\omega_{0}(x)$ restricts the gauge parameters $\varepsilon^{\Omega}(x)$ to the parameters $\varepsilon_{g l}^{\Omega}(x)$ obeying the conditions

$$
\delta \omega_{0}(x)=0 \quad \longrightarrow \quad D_{0} \varepsilon_{g l}^{\Omega}(x)=0, \quad D_{0}:=d+\omega_{0} .
$$

Since $D_{0}^{2}=0, \varepsilon_{g l}^{\Omega}(x)$ is reconstructed (locally) in terms of $\varepsilon_{g l}^{\Omega}\left(x_{0}\right)$ at any $x_{0} . \varepsilon_{g l}^{\Omega}\left(x_{0}\right)$ are the global symmetry parameters of the equation $D_{0} \mathcal{C}(x)=0$.

Alternatively, one can write a solution in the pure gauge form

$$
\omega_{0}(x)=g^{-1}(x) d g(x), \quad \mathcal{C}(x)=g^{-1}(x) \mathcal{C}, \quad \varepsilon_{g l}(x)=g^{-1}(x) \epsilon g(x) .
$$

For $g\left(x_{0}\right)=1$ this gives $\mathcal{C}=\mathcal{C}\left(x_{0}\right)$ and $\epsilon=\varepsilon_{g l}\left(x_{0}\right)$.

\subsection{Massless scalar field unfolded}

Minkowski space is described by a flat Poincaré-connection $\omega(x)=e^{a}(x) P_{a}+\frac{1}{2} \omega^{a b}(x) M_{a b}$. In Cartesian coordinates $e^{a}(x)=\theta^{a}$ and $\omega^{a b}=0$.

Introduce an infinite set of zero-forms, which are traceless symmetric tensors

$$
C_{a_{1} \ldots a_{n}}(x)=C_{\left(a_{1} \ldots a_{n}\right)}(x), \quad \eta^{b c} C_{b c a_{3} \ldots a_{n}}(x)=0 .
$$

The unfolded system of equations equivalent to the Klein-Gordon equation has the form

$$
d C_{a_{1} \ldots a_{n}}(x)=\theta^{b} C_{a_{1} \ldots a_{n} b}(x) .
$$

Since the fields $C_{a_{1} \ldots a_{n}}(x)$ are symmetric while $\theta^{b} \wedge \theta^{c}=-\theta^{c} \wedge \theta^{b}$, the system (5.4) is formally consistent. (Equivalently, the covariant derivative associated with the equation (5.4) rewritten in the form (5.1) is flat.)

The first two equations imply

$$
\partial_{a} C(x)=C_{a}(x), \quad \partial_{a} C_{b}(x)=C_{a b}(x) \longrightarrow C_{a b}(x)=\partial_{a} \partial_{b} C(x) .
$$

Since $C_{a b}(x)$ is traceless this implies

$$
\square C(x)=0 .
$$

All other equations express higher tensor components via higher derivatives of the scalar field

$$
C_{a_{1} \ldots a_{n}}(x)=\partial_{a_{1}} \ldots \partial_{a_{n}} C(x) .
$$

This formula explains the meaning of $C_{a_{1} \ldots a_{n}}(x)$ as spanning a basis of the space of all onmass-shell nontrivial derivatives of $C(x)$. It should be noted that the space of $C_{a_{1} \ldots a_{n}}(x)$ is analogous (in some sense dual) to the space of single-particle states. Via Eq. (5.4) the set of fields $C_{a_{1} \ldots a_{n}}(x)$ at any given $x=x_{0}$ determines $C(x)$ in some neighborhood of $x_{0}$, thus providing a locally complete set of "initial data". 


\subsection{Any $d$}

From the unfolded form of the massless scalar field equations it follows that the conformal HS algebra $h$ in $d$ dimensions is the algebra of linear transformations of the infinite-dimensional space $V$ of various traceless symmetric tensors $C, C_{a}, C_{a b} \ldots$, i.e., $h=g l(V)$. Since the space $V$ is infinite dimensional, such a definition is not fully satisfactory, requiring a more precise definition of the appropriate class of operators. In practice, the idea is that the basis operators of the conformal HS algebra $h$ should reproduce the HS symmetry transformations represented by finite-order differential operators.

A careful definition of $h$ was given by Eastwood in 33] by different methods. As shown in [32], the construction for $d=3$ significantly simplifies in the framework of the spinorial formalism. Since this formulation is most relevant in the context of the $A d S_{4} / C F T_{3} \mathrm{HS}$ holography we explain it in some more detail.

\section{Conformal HS Algebra in $d=3$}

\section{1 $3 d$ multispinors}

Convenience of the language of spinors in $3 d$ theories is due to the following well-known isomorphisms of the $3 d$ Lorentz algebra: $o(2,1) \sim s p(2, R) \sim s l_{2}(R) .3 d$ spinors in Minkowski signature are real

$$
\chi_{\alpha}^{\dagger}=\chi_{\alpha}, \quad \alpha=1,2 .
$$

The $\operatorname{sp}(2, R)$ invariant tensor $\epsilon^{\alpha \beta}=-\epsilon^{\beta \alpha}$ relates lower and upper indices

$$
\chi^{\alpha}=\epsilon^{\alpha \beta} \chi_{\beta}, \quad \chi_{\alpha}=\chi^{\beta} \epsilon_{\beta \alpha} .
$$

Because a two-by-two antisymmetric matrix is unique up to a factor, the antisymmetrization of $3 d$ spinor indices is equivalent to their contraction

$$
A_{\alpha, \beta}-A_{\beta, \alpha}=\epsilon_{\alpha \beta} A_{\gamma}^{\gamma}
$$

As a result, irreducible modules of the Lorentz algebra are represented by various totally symmetric multispinors $A_{\alpha_{1} \ldots \alpha_{n}}$. As a consequence, rank- $k$ traceless symmetric tensors in the tensor notations are equivalent to the rank- $2 k$ totally symmetric multispinors

$$
A_{a_{1} \ldots a_{m}} \sim A_{\alpha_{1} \ldots \alpha_{2 m}}, \quad A_{b a_{3} \ldots a_{m}}^{b}=0 .
$$

(The reader can compare the number of independent components of the both objects).

The explicit relation between the two formalisms is established with the help of the $2 \times 2$ real symmetric matrices $\sigma_{\alpha \beta}^{n}$

$$
A_{\alpha \beta}=\sigma_{\alpha \beta}^{n} A_{n}, \quad \sigma_{\alpha \beta}^{n}=\sigma_{\beta \alpha}^{n} .
$$




\subsection{Spinorial form of $3 d$ massless equations}

In $d=3$, the space $V$ of all traceless symmetric tensors is equivalent to the space of even functions of the commuting spinor variable $y^{\alpha}$

$$
C(y \mid x)=\sum_{n=0}^{\infty} C^{\alpha_{1} \ldots \alpha_{2 n}}(x) y_{\alpha_{1}} \ldots y_{\alpha_{2 n}} .
$$

In these terms, the unfolded equations for a massless scalar take the form

$$
\theta^{\alpha \beta}\left(\frac{\partial}{\partial x^{\alpha \beta}}+\frac{\partial^{2}}{\partial y^{\alpha} \partial y^{\beta}}\right) C(y \mid x)=0
$$

with $C(-y \mid x)=C(y \mid x)$. The same equation with odd $C(-y \mid x)=-C(y \mid x)$ describes a $3 d$ massless spinor field $C_{\alpha}(x)=\left.\frac{\partial}{\partial y^{\alpha}} C(y \mid x)\right|_{y=0} 32$.

\section{$6.33 d$ HS symmetry}

The $3 d$ bosonic conformal HS algebra is the algebra of various differential operators $\epsilon\left(y, \frac{\partial}{\partial y}\right)$ obeying

$$
\epsilon\left(-y,-\frac{\partial}{\partial y}\right)=\epsilon\left(y, \frac{\partial}{\partial y}\right)
$$

The transformation law is

$$
\delta C(y \mid x)=\varepsilon_{g l}\left(y, \frac{\partial}{\partial y} \mid x\right) C(y \mid x)
$$

where

$$
\varepsilon_{g l}\left(y, \frac{\partial}{\partial y} \mid x\right)=\exp \left[-x^{\alpha \beta} \frac{\partial^{2}}{\partial y^{\alpha} \partial y^{\beta}}\right] \epsilon\left(y, \frac{\partial}{\partial y}\right) \exp \left[x^{\alpha \beta} \frac{\partial^{2}}{\partial y^{\alpha} \partial y^{\beta}}\right]
$$

We leave it to the reader to check that this transformation indeed maps a solution of (6.1) to

a solution. For any polynomial $\epsilon\left(y, \frac{\partial}{\partial y}\right), \epsilon_{g l}\left(y, \frac{\partial}{\partial y} \mid x\right)$ is polynomial as well. $\epsilon_{g l}\left(y, \frac{\partial}{\partial y}\right)$ provides the generating function for parameters of the global HS transformations.

The $3 d$ conformal algebra $s p(4) \sim o(3,2)$ is a subalgebra of the HS conformal algebra with the generators

$$
P_{\alpha \beta}=\frac{\partial^{2}}{\partial y^{\alpha} \partial y^{\beta}}, \quad K^{\alpha \beta}=y^{\alpha} y^{\beta}, \quad M_{\alpha \beta}=y_{\alpha} \frac{\partial}{\partial y^{\beta}}+y_{\beta} \frac{\partial}{\partial y^{\alpha}}, \quad D=y^{\alpha} \frac{\partial}{\partial y^{\alpha}}+1
$$

It is not difficult to check how formula (6.2) reproduces the standard conformal transformations for massless scalar and spinor in three dimensions. 


\subsection{Weyl algebra and star product}

The Weyl algebra $A_{n}$ is the associative algebra of polynomials of oscillators $\hat{Y}_{A}$ obeying the commutation relations

$$
\left[\hat{Y}_{A}, \hat{Y}_{B}\right]=2 i C_{A B}, \quad A, B, \ldots=1, \ldots 2 n, \quad C_{A B}=-C_{B A}
$$

with a nondegenerate $C_{A B}$. Taking into account that

$$
\hat{Y}_{A}=\left(\begin{array}{c}
y^{\alpha} \\
i \frac{\partial}{\partial y^{\beta}}
\end{array}\right)
$$

obey the Heisenberg commutation relations (6.5), we conclude that the $3 d$ conformal HS algebra (to be identified with the $A d S_{4}$ HS algebra) is the Lie algebra associated with the even part of the Weyl algebra $A_{2}$.

In practice, it is convenient to replace any operator

$$
\hat{f}(\hat{Y})=\sum_{n=0}^{\infty} \frac{1}{n !} f^{A_{1} \ldots A_{n}} \hat{Y}_{A_{1}} \ldots \hat{Y}_{A_{n}}
$$

with symmetric $f^{A_{1} \ldots A_{n}}$ by its Weyl symbol $f(Y)$ which is the function of commuting variables $Y^{A}\left(Y^{A} Y^{B}=Y^{B} Y^{A}\right)$, that has the same power series expansion

$$
f(Y)=\sum_{n=0}^{\infty} \frac{1}{n !} f^{A_{1} \ldots A_{n}} Y_{A_{1}} \ldots Y_{A_{n}} .
$$

The Weyl star product is defined by the rule that $(f * g)(Y)$ is the symbol of $\hat{f}(\hat{Y}) \hat{g}(\hat{Y})$. In particular, this implies

$$
\left[Y_{A}, Y_{B}\right]_{*}=2 i C_{A B}, \quad[a, b]_{*}=a * b-b * a .
$$

One can also see that

$$
\left\{Y_{A}, f(Y)\right\}_{*}=2 Y_{A} f(Y), \quad\left[Y_{A}, f(Y)\right]_{*}=2 i \frac{\partial}{\partial Y^{A}} f(Y),
$$

where

$$
Y^{A}=C^{A B} Y_{B}
$$

The star product is concisely described by the Weyl-Moyal formula

$$
\left(f_{1} * f_{2}\right)(Y)=f_{1}(Y) \exp \left[i \overleftarrow{\partial^{A}} \overrightarrow{\partial^{B}} C_{A B}\right] f_{2}(Y), \quad \partial^{A}:=\frac{\partial}{\partial Y_{A}}
$$

which can be proven using the Campbell-Hausdorff formula for $\exp J^{A} \hat{Y}_{A}$.

By its definition, the star product (6.6) is associative $(f * g) * h=f *(g * h)$ and regular in the sense that the star product of any two polynomials of $Y$ is a polynomial.

The star product also admits the following useful integral representation

$$
\left(f_{1} * f_{2}\right)(Y)=\frac{1}{\pi^{2 M}} \int d S d T \exp \left(-i S_{A} T_{B} C^{A B}\right) f_{1}(Y+S) f_{2}(Y+T) .
$$




\section{HS Symmetry in $A d S_{4}$}

\subsection{Spinor language in four dimension}

The HS theory in four dimensions is most naturally formulated in terms of two-component spinors which language is closely related to the twistor theory. Here the key fact is that $2 \times 2=4$. Minkowski coordinates are represented by $2 \times 2$ Hermitian matrices

$$
X^{n} \Rightarrow X^{\alpha \dot{\alpha}}=\sum_{n=0}^{3} X^{n} \sigma_{n}^{\alpha \dot{\alpha}}, \quad \sigma_{n}^{\alpha \dot{\alpha}}=\left(I^{\alpha \dot{\alpha}}, \vec{\sigma}^{\alpha \dot{\alpha}}\right)
$$

where $I^{\alpha \dot{\alpha}}$ is the unit matrix and $\vec{\sigma}^{\alpha \dot{\alpha}}$ are Pauli matrices. $\alpha, \beta, \ldots=1,2, \dot{\alpha}, \dot{\beta}, \ldots=1,2$ are two-component spinor indices.

In these terms

$$
\operatorname{det}\left|X^{\alpha \dot{\alpha}}\right|=\left(X^{0}\right)^{2}-\left(X^{1}\right)^{2}-\left(X^{2}\right)^{2}-\left(X^{3}\right)^{2} .
$$

This relation establishes the well-known isomorphism for the four-dimensional Lorentz algebra $s l(2, \mathbb{C}) \sim o(3,1)$.

The dictionary between tensors and multispinors is provided by the $\sigma$-matrices

$$
\sigma_{\alpha \dot{\alpha}}^{a}, \quad \sigma_{\alpha \beta}^{a b}=\sigma_{\alpha \dot{\alpha}}^{[a} \sigma_{\beta}^{b]} \dot{\alpha}, \quad \bar{\sigma}_{\dot{\alpha} \dot{\beta}}^{a b}=\sigma_{\alpha \dot{\alpha}}^{[a} \sigma_{\dot{\beta}}^{b] \alpha},
$$

where the two-component indices are raised and lowered by the two-by-two antisymmetric form $\varepsilon_{\alpha \beta}$,

$$
y^{\alpha}=\varepsilon^{\alpha \beta} y_{\beta}, \quad y_{\alpha}=y^{\beta} \varepsilon_{\beta \alpha}, \quad \varepsilon_{\alpha \gamma} \varepsilon^{\beta \gamma}=\delta_{\alpha}^{\beta}, \quad \varepsilon_{12}=\varepsilon^{12}=1 .
$$

These relations show that a pair of dotted and undotted indices is equivalent to a vector index, while the pairs of symmetrized indices of the same type are equivalent to the secondrank antisymmetric tensors.

\section{2 $\quad A d S_{4}$ HS algebra}

The identification of the $3 d$ conformal HS symmetry with the $A d S_{4}$ HS symmetry implies that the global symmetry of the most symmetric vacuum of the bosonic HS theory is represented by the Lie algebra associated with the even part of the Weyl algebra $A_{2}$. To have $4 d$ Lorentz symmetry manifest, it is most convenient to realize $A_{2}$ as the algebra of mutually conjugate operators $y_{\alpha}$ and $\bar{y}_{\dot{\alpha}}$ that obey the star-product commutation relations

$$
\left[y_{\alpha}, y_{\beta}\right]_{*}=2 i \varepsilon_{\alpha \beta}, \quad\left[\bar{y}_{\dot{\alpha}}, \bar{y}_{\dot{\beta}}\right]_{*}=2 i \varepsilon_{\dot{\alpha} \dot{\beta}} .
$$

Historically, the $A d S_{4}$ HS algebra was originally found in [34] by different methods from the analysis of the HS fields in $A d S_{4}$ while its relation to the Weyl algebra was found in [35. 
This realization is convenient for the analysis of the properties of the HS algebra. Spin- $s$ generators are represented by the homogeneous polynomials $T_{s}(y, \bar{y})$ of degree $2(s-1)$. The commutation relations have the following structure

$$
\left[T_{s_{1}}, T_{s_{2}}\right]=T_{s_{1}+s_{2}-2}+T_{s_{1}+s_{2}-4}+\ldots+T_{\left|s_{1}-s_{2}\right|+2} .
$$

Once a spin $s>2$ appears, the HS algebra contains an infinite tower of higher spins. Indeed, since $\left[T_{s}, T_{s}\right]$ gives rise to $T_{2 s-2}$, further commutators then lead to higher and higher spins. Note also that $\left[T_{s}, T_{s}\right]$ contains the generators $T_{2}$ of the $A d S_{4}$ algebra $o(3,2) \sim s p(4)$.

The HS gauge fields in four dimensions are the one-forms

$$
\omega(Y \mid X)=\sum_{n, m=0}^{\infty} \frac{1}{2 n ! m !} \omega_{\alpha_{1} \ldots \alpha_{n}, \dot{\alpha}_{1} \ldots \dot{\alpha}_{m}}(X) y^{\alpha_{1}} \ldots y^{\alpha_{n}} \bar{y}^{\dot{\alpha}_{1}} \ldots \bar{y}^{\dot{\alpha}_{m}}
$$

where $Y_{A}=\left(y_{\alpha}, \bar{y}_{\dot{\alpha}}\right)$ are commuting spinor variables and $X$ are local coordinates of $A d S_{4}$. The HS curvatures and gauge transformations are

$$
\begin{gathered}
R(Y \mid X)=d \omega(Y \mid X)+\omega(Y \mid X) * \omega(Y \mid X), \\
\delta \omega(Y \mid X)=D \epsilon(Y \mid X)=d \epsilon(Y \mid X)+[\omega(Y \mid X), \epsilon(Y \mid X)]_{*} .
\end{gathered}
$$

The symmetry algebra of a single boundary scalar field called $h u(1,0 \mid 4)$ contains every spin in one copy. Conventional symmetries are associated with spins $s \leq 2$, forming finitedimensional subalgebras of the HS algebra. For example, the maximal finite-dimensional subalgebra of $h u(1,0 \mid 4)$ is $u(1) \oplus o(3,2)$ where $u(1)$ is associated with the unit element of the star-product algebra.

More generally, there are three series of $4 d$ HS superalgebras, namely $h u(n, m \mid 4), h o(n, m \mid 4)$ and husp $(2 n, 2 m \mid 4)$. Spin-one fields of the respective HS theories are the Yang-Mills fields of the Lie groups $G=U(n) \times U(m), O(n) \times O(m)$ and $U s p(2 n) \times U s p(2 m)$, respectively. Fermions belong to the bifundamental modules of the two components of $G$. All odd spins are in the adjoint representation of $G$. Even spins carry the opposite symmetry second rank representation of $G$. Namely, in the $h u(n, m \mid 4)$ HS theories they are still in the adjoint representation of $U(n) \times U(m)$, while in the $h o(n, m \mid 4)$ and $h u s p(2 n, 2 m \mid 4)$ HS theories even spins carry rank-two symmetric representation of $O(n) \times O(m)$ and antisymmetric representation of $U s p(2 n) \times U \operatorname{sp}(2 m)$, respectively. The ho(1,0|4) HS theory is the minimal one only containing even spins $s=0,2,4,6, \ldots$

The HS theories have the important feature that their particle spectrum always contains a colorless graviton and a colorless scalar which are both invariant under the spin-one YangMills internal symmetries. It is interesting to note that the presence of the colorless scalar field in the spectrum, which is a standard ingredient of the modern cosmological models, is one of the predictions of the HS symmetry. 


\section{Free HS fields in four dimension}

\subsection{Vacuum solution}

Whatever form they have, nonlinear HS field equations will be formulated in terms of the HS curvatures. Hence, any connection $\omega(Y \mid X)$ that has zero curvature solves the nonlinear HS equations of motion. Such solutions include in particular the $A d S_{4}$ connection because $A d S_{4}$ is described by the flat gravitational connections of $s p(4)$ which is a subalgebra of the HS algebra.

The $A d S_{4}$ vacuum solution solves the equations

$$
R_{0}=0
$$

for $\omega_{0} \in s p(4) \sim o(3,2)$ that has the form

$$
\omega_{0}(Y \mid X)=\frac{1}{4 i}\left(w^{\alpha \beta}(X) y_{\alpha} y_{\beta}+\bar{w}^{\dot{\alpha} \dot{\beta}}(X) \bar{y}_{\dot{\alpha}} \bar{y}_{\dot{\beta}}+2 \lambda h^{\alpha \dot{\beta}}(X) y_{\alpha} \bar{y}_{\dot{\beta}}\right) .
$$

We leave it to the reader to check that these equations indeed describe $A d S_{4}$.

Fluctuations describe small deviations of all massless fields from the vacuum

$$
\omega=\omega_{0}+\omega_{1}, \quad R_{1}=D_{0} \omega_{1}:=d \omega_{1}+\left[\omega_{0}, \omega_{1}\right]_{*} .
$$

Since we know free massless field equations, we anticipate them to result from the linearization of the full nonlinear system. The key question is in which form the free massless field equations will follow from the full nonlinear system? The appropriate form is provided by the Central on-shell theorem.

\subsubsection{Central on-shell theorem}

The full unfolded system for the free massless fields of all spins can be formulated in terms of the one-form $\omega(Y \mid X)$ and zero-form $C(Y \mid X)$ as follows [36]:

$$
\begin{gathered}
R_{1}(Y \mid X)=\bar{H}^{\dot{\alpha} \dot{\beta}} \frac{\partial^{2}}{\partial \bar{y}^{\alpha} \partial \bar{y}^{\dot{\beta}}} C(0, \bar{y} \mid X)+H^{\alpha \beta} \frac{\partial^{2}}{\partial y^{\alpha} \partial y^{\beta}} C(y, 0 \mid X), \\
\tilde{D}_{0} C(Y \mid X)=0,
\end{gathered}
$$

where

$$
H^{\alpha \beta}=h_{\dot{\alpha}}^{\alpha} \wedge h^{\beta \dot{\alpha}}, \quad \bar{H}^{\dot{\alpha} \dot{\beta}}=h_{\alpha}^{\dot{\alpha}} \wedge h^{\alpha \dot{\beta}}
$$

are the basis two-forms in four dimension,

$$
\begin{gathered}
R_{1}(Y \mid X)=D_{0}^{a d} \omega(Y \mid X), \\
D_{0}^{a d}=D^{L}-\lambda h^{\alpha \dot{\beta}}\left(y_{\alpha} \frac{\partial}{\partial \bar{y}^{\dot{\beta}}}+\frac{\partial}{\partial y^{\alpha}} \bar{y}_{\dot{\beta}}\right), \quad \tilde{D}_{0}=D^{L}+\lambda h^{\alpha \dot{\beta}}\left(y_{\alpha} \bar{y}_{\dot{\beta}}+\frac{\partial^{2}}{\partial y^{\alpha} \partial \bar{y}^{\dot{\beta}}}\right),
\end{gathered}
$$


and the Lorentz covariant derivative is

$$
D^{L} A=d_{X}-\left(\omega^{\alpha \beta} y_{\alpha} \frac{\partial}{\partial y^{\beta}}+\bar{\omega}^{\dot{\alpha} \dot{\beta}} \bar{y}_{\dot{\alpha}} \frac{\partial}{\partial \bar{y}^{\dot{\beta}}}\right) .
$$

Since the system of equations (8.1) and (8.2) contains the exhaustive information about free massless fields, including all their dual formulations, it is called Central on-shell theorem.

The pattern of Eqs. (8.1) and (8.2) is as follows. The gauge fields of different spins are described by the homogeneous polynomials in $Y$

$$
\omega^{s}(\nu y, \nu \bar{y} \mid X)=\nu^{2(s-1)} \omega(y, \bar{y} \mid X) .
$$

The zero-forms associated with the spin $s$ obey

$$
C^{s}\left(\nu y, \nu^{-1} \bar{y} \mid X\right)=\nu^{ \pm 2 s} C(y, \bar{y} \mid X)
$$

This implies that a set of one-forms associated with a massless spin $s$ contains a finite number of components while a set of zero-forms contains an infinite number of components. Altogether, these fields describe an infinite set of spins $s=0,1 / 2,1,3 / 2,2,5 / 2 \ldots$

$$
\omega_{\alpha_{1} \ldots \alpha_{n}, \dot{\beta}_{1} \ldots \dot{\beta}_{m}}^{s}: \quad n+m=2(s-1), \quad C_{\alpha_{1} \ldots \alpha_{n}, \dot{\beta}_{1} \ldots \dot{\beta}_{m}}^{s}: \quad|n-m|=2 s .
$$

The zero-forms $C(Y \mid X)$ encode the gauge invariant HS curvatures and spin-zero matter fields along with all their derivatives that remain non-zero on the dynamical field equations. Dynamical fields include the frame-like fields $\omega_{\alpha_{1} \ldots \alpha_{s-1}, \dot{\beta}_{1} \ldots \dot{\beta}_{s-1}}^{s}$ and the scalar $C(0,0 \mid x)$. The frame-like fields reduce to the Fronsdal fields upon gauge fixing of the Lorentz-like Stueckelberg gauge symmetries in the linearized HS gauge transformation (7.2).

All other fields from the list (8.3) are expressed by Eqs. (8.1) and (8.2) via higher derivatives of the dynamical fields. The derivatives come in the dimensionless combination

$$
\lambda^{-1} \frac{\partial}{\partial x}, \quad \lambda^{2}=-\Lambda
$$

with the inverse radius $\lambda$ of the background $A d S$ space. As a result, the HS interactions, that contain higher derivatives, turn out to be nonanalytic in the cosmological constant $\Lambda$ of the background $A d S$ space.

\subsubsection{Examples}

In the spin-zero sector, the Central on-shell theorem just reproduces the unfolded equations for a scalar field. Indeed, the set of all multispinors $C_{\alpha_{1} \ldots \alpha_{n}, \dot{\beta}_{1} \ldots \dot{\beta}_{n}}^{0}$ with the equal numbers of dotted and undotted spinor indices provides the spinorial realization of the set of all symmetric traceless tensors $C_{a_{1} \ldots a_{n}}, C^{b}{ }_{b a_{3} \ldots a_{n}}=0$ in four dimension.

Leaving the derivation of the Maxwell equations in the spin-one sector to the reader, we consider the case of spin two. Here the gauge fields include the Lorentz connection $\omega_{\alpha \beta}, \bar{\omega}_{\dot{\alpha} \dot{\beta}}$ 
and the frame field $\omega_{\alpha, \dot{\beta}}$. The zero-forms $C_{\alpha_{1} \alpha_{2} \alpha_{3} \alpha_{4}}(X)$ and $\bar{C}_{\dot{\alpha}_{1} \dot{\alpha}_{2} \dot{\alpha}_{3} \dot{\alpha}_{4}}(X)$ describe the Weyl tensor in terms of two-component spinors. Higher components $C_{\alpha_{1} \ldots \alpha_{n}, \dot{\beta}_{1} \ldots \dot{\beta}_{m}}^{s}$ with $|n-m|=4$ describe all its non-trivial derivatives.

Consider first Eq. (8.1). The equation $R_{\alpha, \dot{\beta}}=0$ is the usual zero-torsion condition that expresses the Lorentz connection via the vierbein. The other equations have the form

$$
R_{\alpha \beta}=H^{\gamma \delta} C_{\alpha \beta \gamma \delta}, \quad R_{\dot{\alpha} \dot{\beta}}=\bar{H}^{\dot{\gamma} \dot{\delta}} \bar{C}_{\dot{\alpha} \dot{\beta} \dot{\gamma} \dot{\delta}} .
$$

These imply that a nonzero part of the Riemann tensor belongs to the Weyl tensor. This is equivalent to saying that the Ricci tensor is zero which, in turn, is equivalent to the Einstein equations in the vacuum.

In the tensorial language the same equations read as

$$
R_{\nu \mu}^{a}=0, \quad R_{\nu \mu}^{a b}=e_{\nu}{ }^{c} e_{\mu}{ }^{d} C_{c d}{ }^{a b}, \quad C_{a b,{ }^{b}}{ }^{b}=0 .
$$

This implies the Einstein equations since $R_{\nu \mu}=R_{\nu \rho}{ }^{\rho}{ }_{\mu}=0$. In addition, the system (8.5) implies that $C_{c d,}{ }^{a b}$ coincides with the Weyl tensor.

Analogously, the Central on-shell theorem for higher spins imposes the Fronsdal equations $\mathcal{R}_{\nu_{1} \ldots \nu_{s}}=0$ and expresses the generalized HS Weyl tensors in terms of derivatives of the Fronsdal fields.

\section{$9 \quad$ Nonlinear higher-spin theory}

In this section we briefly summarize the construction of nonlinear HS equations. The reader not interested in technical details is advised to go directly to Section 9.4.

\subsection{Idea of construction}

The idea is to look for nonlinear HS field equations in the form of a nonlinear deformation of the Central on-shell theorem. The first step is to replace the linearized HS curvatures and covariant derivatives by the full non-Abelian ones:

$$
\begin{gathered}
R(y, \bar{y} \mid X)=d \omega(y, \bar{y} \mid X)+\omega(y, \bar{y} \mid X) * \omega(y, \bar{y} \mid X), \\
\tilde{D} C(y, \bar{y} \mid X)=d C(y, \bar{y} \mid X)+\omega(y, \bar{y} \mid X) * C(y, \bar{y} \mid X)-C(y, \bar{y} \mid X) * \omega(y,-\bar{y} \mid X)
\end{gathered}
$$

trying to find a deformation of the form

$$
\begin{gathered}
R(Y \mid X)=\bar{H}^{\dot{\alpha} \dot{\beta}} \frac{\partial^{2}}{\partial \bar{y}^{\dot{\alpha}} \partial \bar{y}^{\dot{\beta}}} C(0, \bar{y} \mid X)+H^{\alpha \beta} \frac{\partial^{2}}{\partial y^{\alpha} \partial y^{\beta}} C(y, 0 \mid X)+\ldots, \\
\tilde{D} C(Y \mid X)+\ldots=0
\end{gathered}
$$

where further nonlinear corrections have to be determined from the formal consistency of the HS equations. Having the form of the generalized Bianchi identities, the consistency of the HS equations also guarantees their gauge invariance. 
Field equations of such a form are called unfolded which means that all dynamical fields are differential forms and that the exterior derivative of any field is expressed via the exterior product of the fields themselves. As discussed in some more detail in Section 10, this form of dynamical equations is useful in many respects.

Being possible in a few first orders, the straightforward construction of the nonlinear deformation quickly gets complicated. The trick is to find a larger algebra $g^{\prime}$ such that an appropriate substitution

$$
\omega \rightarrow W=\omega+\omega C+\omega C^{2}+\ldots
$$

into $W \in g^{\prime}$ reconstructs nonlinear equations via the flatness condition

$$
d W+W \wedge W=0
$$

The problem is to find appropriate restrictions on $W$ that reconstruct the nonlinear HS equations in all orders.

This is achieved via the doubling of spinors

$$
\omega(Y \mid X) \longrightarrow W(Z ; Y \mid X), \quad C(Y \mid X) \longrightarrow B(Z ; Y \mid X)
$$

accompanied by the equations that determine the dependence on the additional spinorial variables $Z^{A}$ in terms of the "initial data"

$$
\omega(Y \mid X)=W(0 ; Y \mid X), \quad C(Y \mid X)=B(0 ; Y \mid X)
$$

where $\omega(Y \mid X)$ and $C(Y \mid X)$ are the HS fields of the Central on-shell theorem. To rewrite the evolution along the additional variables $Z^{A}$ covariantly, it is useful to introduce a connection $S(Z, Y \mid X)=d Z^{A} S_{A}$ in the $Z^{A}$-space.

\subsection{HS star product}

Nonlinear HS field equations are formulated in terms of the specific star product

$$
(f * g)(Z ; Y)=\frac{1}{(2 \pi)^{4}} \int d^{4} U d^{4} V \exp \left[i U^{A} V^{B} C_{A B}\right] f(Z+U ; Y+U) g(Z-V ; Y+V)
$$

where $C_{A B}=\left(\varepsilon_{\alpha \beta}, \bar{\varepsilon}_{\dot{\alpha} \dot{\beta}}\right)$ is the $4 d$ charge conjugation matrix and $U^{A}, V^{B}$ are real integration variables. The normalization is such that 1 is a unit element of the star-product algebra, i.e., $f * 1=1 * f=f$. The star product (9.1) is associative and provides a particular realization of the Weyl algebra since

$$
\left[Y_{A}, Y_{B}\right]_{*}=-\left[Z_{A}, Z_{B}\right]_{*}=2 i C_{A B} \quad\left[Y_{A}, Z_{B}\right]_{*}=0
$$

It results from the normal ordering with respect to the elements

$$
b_{A}=\frac{1}{2 i}\left(Y_{A}-Z_{A}\right), \quad a_{A}=\frac{1}{2}\left(Y_{A}+Z_{A}\right),
$$


which satisfy

$$
\left[a_{A}, a_{B}\right]_{*}=\left[b_{A}, b_{B}\right]_{*}=0, \quad\left[a_{A}, b_{B}\right]_{*}=C_{A B}
$$

and can be interpreted as creation and annihilation operators. In fact, the star product (9.1) describes the normal ordering with respect to the oscillators $a_{A}$ and $b_{A}$ as is most evident from the following consequences of (9.1):

$$
b_{A} * f(b, a)=b_{A} f(b, a), \quad f(b, a) * a_{A}=f(b, a) a_{A} .
$$

An important property of the star product (9.1) is that it admits the inner Klein operator

$$
\Upsilon=\exp i Z_{A} Y^{A}
$$

which behaves as $(-1)^{N}$, where $N$ is the spinor number operator. One can easily see that

$$
\begin{gathered}
\Upsilon * \Upsilon=1, \\
\Upsilon * f(Z ; Y)=f(-Z ;-Y) * \Upsilon
\end{gathered}
$$

and

$$
(\Upsilon * f)(Z ; Y)=\exp i Z_{A} Y^{A} f(Y ; Z) .
$$

With respect to the decomposition of Majorana spinors into two-component spinors, $Y_{A}=\left(y_{\alpha}, \bar{y}_{\dot{\alpha}}\right), \bar{y}_{\dot{\alpha}}=\left(y_{\alpha}\right)^{\dagger}$, the left and right inner Klein operators

$$
\kappa=\exp i z_{\alpha} y^{\alpha}, \quad \bar{\kappa}=\exp i \bar{z}_{\dot{\alpha}} \bar{y}^{\dot{\alpha}}
$$

act analogously on the undotted and dotted spinors, respectively,

$$
\begin{gathered}
\kappa * f(z, \bar{z} ; y, \bar{y})=f(-z, \bar{z} ;-y, \bar{y}) * \kappa, \quad \bar{\kappa} * f(z, \bar{z} ; y, \bar{y})=f(z,-\bar{z} ; y,-\bar{y}) * \bar{\kappa}, \\
\kappa * \kappa=\bar{\kappa} * \bar{\kappa}=1, \quad \kappa * \bar{\kappa}=\bar{\kappa} * \kappa .
\end{gathered}
$$

\subsection{The full nonlinear system}

As shown in [37], the equations of motion of the four-dimensional HS theory can be formulated in terms of the three types of fields

$$
W=d X^{\nu} W_{\nu}(Z, Y ; K \mid X), \quad S=d Z^{A} S_{A}(Z, Y ; K \mid X), \quad B(Z, Y ; K \mid X) .
$$

The fields $W$ and $S$ are, respectively, one-forms in the four-dimensional space-time with the coordinates $X^{\nu}$ and spinor space with the coordinates $Z_{A}$. The spinorial variables $Z_{A}$ and $Y_{A}$ are commuting while $d Z_{A}$ are anticommuting differentials

$$
Z_{A} Z_{B}=Z_{B} Z_{A}, \quad Y_{A} Y_{B}=Y_{B} Y_{A}, \quad Z_{A} Y_{B}=Y_{B} Z_{A}, \quad d Z_{A} d Z_{B}=-d Z_{B} d Z_{A} .
$$

$d Z_{A}$ commute with $Z_{B}$ and $Y_{B}$ but anticommute with the anticommuting space-time differentials $d X^{\nu}$

$$
d X_{\nu} d X_{\mu}=-d X_{\mu} d X_{\nu}, \quad d Z_{A} d X_{\nu}=-d X_{\nu} d Z_{A} .
$$


$K$ denotes a pair of Klein operators $K=(k, \bar{k})$ that obey the relations

$$
\begin{gathered}
k^{2}=\bar{k}^{2}=1, \quad k \bar{k}=\bar{k} k, \\
k w_{\alpha}=-w_{\alpha} k, \quad \bar{k} w_{\alpha}=w_{\alpha} \bar{k}, \quad k \bar{w}_{\dot{\alpha}}=\bar{w}_{\dot{\alpha}} k, \quad \bar{k}_{\dot{\alpha}}=-\bar{w}_{\dot{\alpha}} \bar{k}
\end{gathered}
$$

for $w_{\alpha}=\left(d z_{\alpha}, z_{\alpha}, y_{\alpha}\right), \bar{w}_{\dot{\alpha}}=\left(d \bar{z}_{\dot{\alpha}}, \bar{z}_{\dot{\alpha}}, \bar{y}_{\dot{\alpha}}\right)$. The important difference between $(k, \bar{k})$ and $(\kappa, \bar{\kappa})$ (9.3) is that the former anticommute with the respective differentials $d z^{\alpha}$ and $d \bar{z}^{\dot{\alpha}}$ while the latter commute.

The system of nonlinear HS equations in $d=4$ reads as [37]

$$
\begin{gathered}
d W=W * W, \\
d B=W * B-B * W, \\
d S=W * S-S * W, \\
S * B=B * S, \\
S * S=-i\left(d Z^{A} d Z_{A}+d z^{\alpha} d z_{\alpha} F_{*}(B) * k \kappa+d \bar{z}^{\dot{\alpha}} d \bar{z}_{\dot{\alpha}} \bar{F}_{*}(B) * \bar{k} \bar{\kappa}\right) .
\end{gathered}
$$

$F_{*}(B)$ in Eq. (9.10) is some star-product function of the field $B$. The simplest case of the linear functions

$$
F_{*}(B)=\eta B, \quad \bar{F}_{*}(B)=\bar{\eta} B,
$$

where $\eta$ is some phase factor (its absolute value can be absorbed into a redefinition of $B$ ), leads to the class of pairwise nonequivalent nonlinear HS theories. The cases of $\eta=1$ and $\eta=\exp \frac{i \pi}{2}$ are particularly interesting, corresponding to the so called $A$ and $B$ HS models. These two cases are distinguished by the property that they respect parity [25].

Expanding all fields in powers of $k$ and $\bar{k}$ we obtain for $U=W, S, B$

$$
U(Z, Y ; K \mid X)=\sum_{i, j=0}^{1} k^{i} \bar{k}^{j} U_{i j}(Z, Y \mid X) .
$$

Since the relations (9.4) and (9.5) are invariant under the reflections $k \rightarrow-k$ and $\bar{k} \rightarrow-\bar{k}$, and taking into account that the r.h.s. of Eq. (9.10) contains $k$ and $\bar{k}$ explicitly, it follows that the system (9.6)-(9.10) is invariant under the following involutive map

$$
\begin{aligned}
\tau(W(Y, Z ; K \mid X))= & W(Y, Z ;-K \mid X), \quad \tau(S(Y, Z ; K \mid X))=S(Y, Z ;-K \mid X), \\
& \tau(B(Y, Z ; K \mid X))=-B(Y, Z ;-K \mid X) .
\end{aligned}
$$

As a result, the full system of fields decomposes into $\tau$-even and $\tau$-odd fields. Clearly, the $\tau$-even fields form a subsystem of the full system while the $\tau$-odd fields can be consistently truncated away. This truncation is applied in most of applications. The dynamical role of the $\tau$-even and $\tau$-odd fields is different.

The $\tau$-even fields we call dynamical since they describe massless fields of various spins. These are $W_{i i}^{d y n}, S_{i i}^{d y n}$ and $B_{i 1-i}^{d y n}$. Each of them appears in two copies because $i=1,2$. As shown in [38], this doubling is inevitable in presence of fermions. 
Each member of the infinite set of the $\tau$-odd fields describes at most a finite number of degrees of freedom. To stress that they carry no local degrees of freedom, they were called auxiliary in [37]. It is also appropriate to call them moduli fields since the finite number of degrees of freedom carried by each of these fields can be interpreted as a kind of coupling constants of the theory. In particular, this was demonstrated in [39] where it was shown that the mass parameter of the matter fields in the $3 d$ HS theory results from a non-zero vacuum value of one of the moduli fields. The moduli fields include $W_{i 1-i}^{\text {mod }}, S_{i 1-i}^{\text {mod }}$ and $B_{i i}^{\text {mod }}$. Truncating away the moduli fields greatly reduces the moduli space of the theory. In particular, the moduli responsible for the massive boundary deformation can be argued to belong to this sector.

The perturbative analysis performed around the following vacuum solution

$$
B_{0}=0, \quad S_{0}=d Z^{A} Z_{A}, \quad W_{0}=\frac{1}{2} \omega_{0}^{\mu \nu}(X) Y_{\mu} Y_{\nu},
$$

where $W_{0}$ obeys

$$
d W_{0}+W_{0} \star W_{0}=0
$$

so that $\omega_{0}^{\mu \nu}(X)$ describes the $A d S_{4}$, reproduces the Central on-shell theorem in the firstorder [37]. This means that the nonlinear system (9.6)-(9.10) indeed provides a nonlinear deformation of the free equations of massless fields of all spins. Note that the specific form of the star product (9.1) is crucial for this analysis.

The HS equations exhibit manifest gauge invariance under the gauge transformations

$$
\delta W=d \varepsilon+[W, \varepsilon]_{*}, \quad \delta S=[S, \varepsilon]_{*}, \quad \delta B=[B, \varepsilon]_{*}, \quad \varepsilon=\varepsilon(Z ; Y ; K \mid X) .
$$

The nonlinear HS equations are formally consistent and regular: perturbatively, there are no divergences due to star products of the non-polynomial elements resulting from the inner Klein operators $\kappa$ and $\bar{\kappa}$ [40].

\subsection{Properties of HS interactions}

Let us briefly discuss some of the most important properties of the nonlinear HS equations.

First of all, HS interactions contain higher derivatives. This property is closely related to nonanaliticity of the HS interactions in the cosmological constant $\Lambda=-\rho^{-2}$ which appears in the dimensionless combination $\rho \partial$ where $\rho$ is the $A d S$ radius while $\partial$ denotes the space-time derivative. This has the effect that background HS gauge fields contribute to the higherderivative terms in the evolution equations. As a result, the evolution is determined mostly by the HS fields rather than by the metric. This provides the realization of the anticipated property that Riemannian geometry is not an appropriate tool in the HS theory.

In the HS theory, HS fields source lower-spin fields in particular via the $\omega * \omega$-like terms. Other way around, lower-spin fields source HS fields via the $C^{2}$ terms. In particular, gravity sources the HS fields and vise-versa. Among other things this implies that the Einstein gravity cannot be obtained as a consistent truncation of the HS theory. 
A remarkable feature of the HS equations is that their nontrivial part is only represented by Eq. (9.10) which does not contain the space-time derivative $d$. This suggests that not only Riemannian geometry but even usual coordinates do not play a fundamental role in the system. In fact, this is a general property of unfolded dynamical equations the particular case of which is represented by the nonlinear equations (9.6)-(9.10).

\section{Unfolded Dynamics}

\subsection{General setup}

The unfolded form of dynamical equations provides a covariant generalization of the firstorder form of differential equations

$$
\dot{q}^{i}(t)=\varphi^{i}(q(t)),
$$

which is convenient in many respects. In particular, initial values can be given in terms of the values of variables $q^{i}\left(t_{0}\right)$ at any given point $t_{0}$. As a result, in the first-order formulation, the number of degrees of freedom equals to the number of dynamical variables.

Unfolded dynamics is a multidimensional generalization achieved via the replacement of the time derivative by the de Rham derivative

$$
\frac{\partial}{\partial t} \rightarrow d=\theta^{\nu} \partial_{\nu}
$$

and the dynamical variables $q^{i}$ by a set of differential forms

$$
q^{i}(t) \rightarrow W^{\Omega}(\theta, x)=\theta^{\nu_{1}} \ldots \theta^{\nu_{p}} W_{\nu_{1} \ldots \nu_{p}}^{\Omega}(x)
$$

to reformulate a system of partial differential equations in the first-order covariant form

$$
d W^{\Omega}(\theta, x)=G^{\Omega}(W(\theta, x)) .
$$

Here $G^{\Omega}(W)$ are some functions of the "supercoordinates" $W^{\Omega}$

$$
G^{\Omega}(W)=\sum_{n} f^{\Omega}{ }_{\Lambda_{1} \ldots \Lambda_{n}} W^{\Lambda_{1}} \ldots W^{\Lambda_{n}}
$$

Since $d^{2}=0$, at $d>1$ the functions $G^{\Lambda}(W)$ cannot be arbitrary but have to obey the compatibility conditions

$$
G^{\Lambda}(W) \frac{\partial G^{\Omega}(W)}{\partial W^{\Lambda}} \equiv 0
$$

(Recall that all products of the differential forms $W(\theta, x)$ are the wedge products due to anticommutativity of $\theta^{\nu}$.) Let us stress that these are conditions on the functions $G^{\Lambda}(W)$ rather than on $W$. 
The idea of the unfolded formulation was put forward in the paper [40] where it was realized that the full system of nonlinear equations can be searched in the form (10.1) as a deformation of the Central on-shell theorem.

As a consequence of the compatibility conditions (10.2) the system (10.1) is manifestly invariant under the gauge transformation

$$
\delta W^{\Omega}=d \varepsilon^{\Omega}+\varepsilon^{\Lambda} \frac{\partial G^{\Omega}(W)}{\partial W^{\Lambda}},
$$

where the gauge parameter $\varepsilon^{\Omega}(x)$ is a $\left(p_{\Omega}-1\right)$-form if $W^{\Omega}$ is a $p_{\Omega}$-form. Strictly speaking, this is true for the class of universal unfolded systems in which the compatibility conditions (10.2) hold independently of the dimension $d$ of space-time, i.e., (10.2) should be true disregarding the fact that any $(d+1)$-form is zero. Let us stress that all unfolded systems, which appear in HS theories including those considered in these lectures, are universal.

The unfolded formulation can be applied to the description of invariant functionals of the system in question. Here it is useful to distinguish between the off-shell and on-shell unfolded dynamical systems.

As demonstrated in Section 5.2, most of the relations contained in unfolded equations impose constraints expressing some new fields in terms of derivatives of the old ones. In the off-shell case the unfolded equations just express all fields in terms of derivatives of some ground fields, imposing no differential restrictions on the latter. In the scalar-field example of Section 5.2, to make the system off-shell one should relax the tracelessness condition in (5.3). In this case, the pattern of the unfolded system (5.4) is given by the set of constraints (5.6) which express the higher tensors $C_{a_{1} \ldots a_{n}}(x)$ via derivatives of the ground scalar field $C(x)$. The on-shell unfolded equations not only express all fields in terms of derivatives of the ground fields, but also impose differential restrictions on the latter. In the scalar-field example this is the Klein-Gordon equation (5.5).

As shown in [41, the variety of invariant functionals associated with the unfolded equations (10.1) is described by the cohomology of the operator

$$
Q=G^{\Omega} \frac{\partial}{\partial W^{\Omega}}
$$

which obeys

$$
Q^{2}=0
$$

as a consequence of (10.2). By virtue of (10.1), $Q$-closed $p$-form functions $L_{p}(W)$ are $d$-closed, giving rise to the gauge invariant functionals

$$
S=\int_{\Sigma^{p}} L_{p}
$$

In the off-shell case they can be used to construct invariant action functionals while in the on-shell case they describe conserved charges. (For more detail and examples see [41.) Also, in the on-shell case, $S$ can play a róle of the Hamilton-Jacobi action which becomes a functional of boundary conditions in the context of holographic duality.t

\footnotetext{
${ }^{1}$ I am grateful to Ioannis Papadimitriou for the stimulating discussion of this point.
} 


\subsection{Properties}

The unfolded formulation of partial differential equations has a number of remarkable properties.

- First of all, it has general applicability: every system of partial differential equations can be reformulated in the unfolded form.

- Due to using the exterior algebra formalism, the system is invariant under diffeomorphisms, being coordinate independent.

- Interactions can be understood as nonlinear deformations of $G^{\Omega}(W)$.

- Degrees of freedom are represented by the subset of zero-forms $C^{I}\left(x_{0}\right) \in\left\{W^{\Omega}\left(x_{0}\right)\right\}$ at any $x=x_{0}$. This is analogous to the fact that $q^{i}\left(t_{0}\right)$ describe degrees of freedom in the first-order form of ordinary differential equations. The zero-forms $C^{I}\left(x_{0}\right)$ realize an infinite-dimensional module dual to the space of single-particle states of the system. In the HS theory it is realized as a space of functions of auxiliary variables like $C\left(y, \bar{y} \mid x_{0}\right)$. This space is an analogue of the phase space in the Hamiltonian approach.

- It is worth to mention that the same property of the unfolded dynamics provides a tool to control unitarity in presence of higher derivatives via the requirement that the space of zero-forms like $C(y, \bar{y})$ admits a positive-definite norm preserved by the unfolded equations in question.

The above list of remarkable properties of the unfolded formulation is far from being complete. In particular, the unfolded formulation admits a nice interpretation in terms of Lie algebra cohomology (for more detail see [42]), $L_{\infty}$ algebra [43], $Q$-manifolds and many more (for more detail see e.g., [41, 44] and references therein). The most striking feature of this formulation is however that it makes it possible to describe one and the same dynamical system in space-times of different dimensions.

\section{Space-time metamorphoses}

Unfolded dynamics exhibits independence of the "world-volume" space-time with coordinates $x$. Instead, geometry is encoded by the functions $G^{\Omega}(W)$ in the "target space" of fields $W^{\Omega}$. Indeed, the universal unfolded equations make sense in any space-time independently of a particular realization of the de Rham derivative $d$. For instance one can extend space time by adding additional coordinates $z$

$$
d W^{\Omega}(x)=G^{\Omega}(W(x)), \quad x \rightarrow X=(x, z), \quad d_{x} \rightarrow d_{X}=d_{x}+d_{z}, \quad d_{z}=d z^{u} \frac{\partial}{\partial z^{u}} .
$$

The unfolded equations reconstruct the $X$-dependence in terms of values of the fields $W^{\Omega}\left(X_{0}\right)=W^{\Omega}\left(x_{0}, z_{0}\right)$ at any $X_{0}$. Clearly, to take $W^{\Omega}\left(x_{0}, z_{0}\right)$ in space $M_{X}$ with coordinates $X_{0}$ is the same as to take $W^{\Omega}\left(x_{0}\right)$ in the space $M_{x} \subset M_{X}$ with coordinates $x$. 
The problem becomes most interesting provided that there is a nontrivial vacuum connection along the additional coordinates $z$. This is in particular the case of $A d S / C F T$ correspondence where the conformal flat connection at the boundary is extended to the flat $A d S$ connection in the bulk with $z$ being a radial coordinate of the Poincaré type.

Generally, the unfolding can be interpreted as some sort of a covariant twistor transform

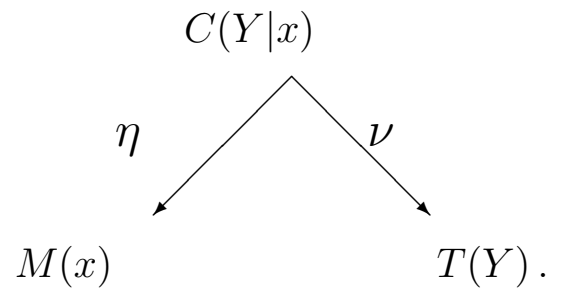

Here $W^{\Omega}(Y \mid x)$ are functions on the "correspondence space" $C$ with local coordinates $Y, x$. The space-time $M$ has local coordinates $x$. The twistor space $T$ has local coordinates $Y$.

The unfolded equations reconstruct the dependence of $W^{\Omega}(Y \mid x)$ on $x$ in terms of the function $W^{\Omega}\left(Y \mid x_{0}\right)$ on $T$ at some fixed $x_{0}$. The restriction of $W^{\Omega}(Y \mid x)$ or some its $Y$ derivatives to $Y=0$ gives dynamical fields $\omega(x)$ in $M$ which, in the on-shell case, solve their dynamical field equations. Hence, similarly to the Penrose transform (see [45] and references therein), unfolded equations map functions on $T$ to solutions of the dynamical field equations in $M$.

In these terms, the holographic duality can be interpreted as the duality between different space-times $M$ that can be associated with the same twistor space. This phenomenon has a number of interesting realizations.

\section{$11.1 \quad A d S_{4} / C F T_{3}$ HS holography}

The $A d S_{4} / C F T_{3}$ HS holography [22] relates the HS gauge theory in $A d S_{4}$ to the quantum theory of conformal currents in three dimensions. To see how it works, let us first discuss the unfolded equations for free massless fields and currents on the $3 d$ boundary.

The unfolded equations for conformal massless fields in three dimensions are [32, 46]

$$
\left(\frac{\partial}{\partial x^{\alpha \beta}} \pm i \frac{\partial^{2}}{\partial y^{\alpha} \partial y^{\beta}}\right) C_{j}^{ \pm}(y \mid x)=0, \quad \alpha, \beta=1,2, \quad j=1, \ldots \mathcal{N}
$$

The equations for $3 d$ conformal conserved currents have the form of rank-two equations [47]

$$
\left\{\frac{\partial}{\partial x^{\alpha \beta}}-\frac{\partial^{2}}{\partial y^{(\alpha} \partial u^{\beta)}}\right\} J(u, y \mid x)=0 \text {. }
$$

$J(u, y \mid x)$ contains all $3 d$ HS currents along with their derivatives.

Elementary $3 d$ conformal currents, which are conformal primaries, contain currents of all spins

$$
J(u, 0 \mid x)=\sum_{2 s=0}^{\infty} u^{\alpha_{1}} \ldots u^{\alpha_{2 s}} J_{\alpha_{1} \ldots \alpha_{2 s}}(x), \quad \tilde{J}(0, y \mid x)=\sum_{2 s=0}^{\infty} y^{\alpha_{1}} \ldots y^{\alpha_{2 s}} \tilde{J}_{\alpha_{1} \ldots \alpha_{2 s}}(x)
$$


along with the additional scalar current

$$
J^{a s y m}(u, y \mid x)=u_{\alpha} y^{\alpha} J^{a s y m}(x) .
$$

Their conformal dimensions are

$$
\Delta J_{\alpha_{1} \ldots \alpha_{2 s}}(x)=\Delta \tilde{J}_{\alpha_{1} \ldots \alpha_{2 s}}(x)=s+1 \quad \Delta J^{a s y m}(x)=2 .
$$

The unfolded equations express all other components of $J(u, y \mid x)$ in terms of derivatives of the primaries, also imposing the differential equations on the primaries, which are just the conservation conditions

$$
\frac{\partial}{\partial x^{\alpha \beta}} \frac{\partial^{2}}{\partial u_{\alpha} \partial u_{\beta}} J(u, 0 \mid x)=0, \quad \frac{\partial}{\partial x^{\alpha \beta}} \frac{\partial^{2}}{\partial y_{\alpha} \partial y_{\beta}} \tilde{J}(0, y \mid x)=0
$$

for all currents except for the scalar ones that do not obey any differential equations.

The rank-two equation is obeyed by

$$
J(u, y \mid x)=\sum_{i=1}^{\mathcal{N}} C_{i}^{-}(u+y \mid x) C_{i}^{+}(y-u \mid x) .
$$

This simple formula gives the explicit realization of the HS conformal conserved currents in terms of bilinear combinations of derivatives of free massless fields in three dimensions.

Generally, the rank-two fields and, hence conserved currents, can be interpreted as bilocal fields in the twistor space. In this respect they are somewhat analogous to space-time bi-local fields also used for the description of currents (see e.g 48, 49 and references therein).

To relate $3 d$ currents to $4 d$ massless fields it remains to extend the $3 d$ current equation to the $4 d$ massless equations. This is easy to achieve in the unfolded dynamics via the extension of the $3 d$ coordinates $x^{\alpha \beta}$ to the $4 d$ coordinates $X^{\alpha \dot{\beta}}$, extending the $3 d$ equations to

$$
\left(\frac{\partial}{\partial X^{\alpha \dot{\alpha}}}+\frac{\partial^{2}}{\partial y^{\alpha} \partial \bar{y}^{\dot{\beta}}}\right) C(y, \bar{y} \mid X)=0 .
$$

These are just the free unfolded equations for $4 d$ massless fields of all spins in Minkowski space, i.e., at $\Lambda=0$.

The analysis in $A d S_{4}$, which is also simple, is performed analogously. In this case, $x^{\alpha \beta}=\frac{1}{2}\left(X^{\alpha \beta}+X^{\beta \alpha}\right)$ are boundary coordinates, while $z^{-1}=X^{\alpha \beta} \epsilon_{\alpha \beta}$ is the radial coordinate. (For more detail see [29].) At the non-linear level, the full HS theory in $A d S_{4}$ turns out to be equivalent to the theory of $3 d$ currents of all spins interacting through conformal HS gauge fields 29.

\section{$11.2 s p(8)$ invariant setup}

Another example of the application of unfolded dynamics is related to the $s p(8)$ extension of conformal symmetry in the theory of massless fields in four dimensions. As was shown 
by Fronsdal [50], the tower of all $4 d$ massless fields is $s p(8)$ symmetric. The $s p(8)$ symmetry extends conformal symmetry $s u(2,2) \subset s p(8)$ that acts on every massless fields. The generators in $s p(8) / s u(2,2)$ mix fields of different spins in the tower of massless fields of all spins $0 \leq s<\infty$.

Indeed, equations (6.2), that describe gauge invariant combinations of massless fields, are covariant constancy conditions for 0 -forms $C(y, \bar{y} \mid x)$ valued in the space of functions of spinor variables $y_{\alpha}$ and $\bar{y}_{\dot{\alpha}}$. Hence, symmetries of these equations contain $s p(8)$ realized by bilinears (6.4) with indices $\alpha$ taking 4 values.

Fronsdal has shown that the space-time $\mathcal{M}_{4}$ appropriate for geometric realization of $S p(8)$ is ten-dimensional with local coordinates $X^{A B}=X^{B A}$, where $A=(\alpha, \dot{\alpha})=1,2,3,4$. Applying the construction of Section 10, it is easy to derive the equations for massless fields in $\mathcal{M}_{4}$.

\subsubsection{From four to ten}

Indeed, unfolded $4 d$ massless equations can be easily uplifted to $\mathcal{M}_{4}$ as follows 46 :

$$
d X^{A B}\left(\frac{\partial}{\partial X^{A B}}+\frac{\partial^{2}}{\partial Y^{A} \partial Y^{B}}\right) C(Y \mid X)=0, \quad A, B=1, \ldots 4
$$

where, for the sake of simplicity, the massless equations are presented in the Cartesian-like coordinates. Note that to obtain the proper $\lambda \rightarrow 0$ limit from Eqs. (6.2), it is necessary to rescale the spinor variables

$$
y_{\alpha} \rightarrow \lambda^{\frac{1}{2}} y_{\alpha}, \quad \bar{y}_{\dot{\alpha}} \rightarrow \lambda^{\frac{1}{2}} \bar{y}_{\dot{\alpha}}
$$

before taking the limit.

If the indices $A, B$ take just two values, equations (11.3) describe $3 d$ massless fields invariant under $S p(4)$ which is the $3 d$ conformal group [32, 46].

By the general argument in the beginning of Section 11, equations (11.3) describe the same dynamics as the original massless field equations in $4 d$ Minkowski space because they consist of the usual $4 d$ equations (11.2) for the coordinates $X^{\alpha \dot{\beta}}$ supplemented with the equations describing the evolution along the additional spinning coordinates $X^{\alpha \beta}$ and $X^{\dot{\alpha} \dot{\beta}}$. The key question is what are independent dynamical variables in $\mathcal{M}_{4}$ ? From (11.3) it is clear that these are the fields $C(0 \mid X)$ and $Y^{A} C_{A}(0 \mid X)$. Indeed, all other components of $C(Y \mid X)$ are expressed by Eq. (11.3) via $X$-derivatives of $C(0 \mid X)$ and $Y^{A} C_{A}(0 \mid X)$. It turns out that $C(0 \mid X)$ describes all $4 d$ massless fields of integer spins while $C_{A}(0 \mid X)$ describes all $4 d$ massless fields of half-integer spins. So, $C(0 \mid X)$ and $C_{A}(0 \mid X)$ serve as certain hyperfields for the HS multiplets.

The nontrivial field equations in $\mathcal{M}_{4}$ are 46

$$
\left(\frac{\partial^{2}}{\partial X^{A B} \partial X^{C D}}-\frac{\partial^{2}}{\partial X^{C B} \partial X^{A D}}\right) C(X)=0
$$

for bosons and

$$
\left(\frac{\partial}{\partial X^{A B}} C_{C}(X)-\frac{\partial}{\partial X^{C B}} C_{A}(X)\right)=0
$$


for fermions. These equations are interesting in many respects. First of all, they are overdetermined. This is what makes it possible to describe the four-dimensional massless fields by virtue of differential equations in the ten-dimensional space $\mathcal{M}_{4}$. Another interesting feature is that equations (11.5) and (11.6) contain no index contraction and hence no metric tensor.

\subsubsection{From ten to four}

It is instructive to see how the usual space-time picture re-appears from the ten-dimensional one. Remarkably, in this setup, the conventional four-dimensional picture results from the identification of a concept of local event simultaneously with the metric tensor. Referring for more detail of the derivation to the original paper [51], we just summarize the final results.

Time in $\mathcal{M}_{M}$ is a parameter $t$ along a time-like direction in $\mathcal{M}_{4}$ represented by any positive-definite matrix $T^{A B}$

$$
X^{A B}=T^{A B} t .
$$

Usual space in $\mathcal{M}_{M}$ is identified with the space of local events at a given time. Coordinates of the space of local events $x^{n}$ are required to have the property that the differential equations in question admit "initial data" localized at any point of pace-time, i.e., represented by the $\delta$-functions $\delta\left(x^{n}-x_{0}^{n}\right)$ with various $x_{0}^{n}$. Since the system of equations in question is overdetermined, the analysis of this issue is not quite trivial. The final result is [51 that, for Eqs. (11.5),(11.6), the space of local events in $\mathcal{M}_{4}$ is represented by a Clifford algebra with

$$
X^{A B}=x^{n} \gamma_{n}^{A} T^{B C}
$$

formed by matrices $\gamma_{n B}^{A}$ that obey

$$
\left\{\gamma_{n}, \gamma_{m}\right\}=2 g_{n m}
$$

where $g_{n m}$ is the spatial metric tensor of $R^{3}$.

Thus, the three-dimensional space of the $4 d$ Minkowski space appears as the space $R^{3}$ of local events. In this analysis, the metric tensor appears just after the identification of coordinates that parametrize local events with the generators of the Clifford algebra. In a certain sense, this construction is opposite to the original Dirac's construction where the $\gamma$-matrices were introduced as a square root of the metric tensor. Here, the metric tensor appears from the definition of the $\gamma$-matrices that represent local events.

Analogous analysis can be performed in some other dimensions. In particular in [52, 51, 53. it was shown that equations (11.3) at $M=2,4,8,16$ describe free conformal fields of all spins in $d=3,4,6,10$.

It should be noted that different $s p(2 M)$-symmetric field equations in the same space $\mathcal{M}_{M}$, like e.g. the higher-rank equations of [47], have spaces of local events of different dimensions. The resulting picture is somewhat analogous to the brane picture in String Theory allowing the co-existence of objects of different dimensions in the same space. The difference is however that the "HS branes" in the $s p(2 M)$ setup are not localized as a particular surface embedded into $\mathcal{M}_{M}$. Instead, different choices of a representative surface 
is a matter of the gauge choice. This example gives another manifestation of the general property that higher symmetries may affect such fundamental concepts as local event and space-time dimension.

\section{HS theory and quantum mechanics}

Classical HS theory has several interesting links with quantum mechanics.

One is that unfolded dynamics in the spinor (twistor) formulation distinguishes between positive and negative frequencies

$$
\left(\frac{\partial}{\partial X^{A B}} \pm i \frac{\partial^{2}}{\partial Y^{A} \partial Y^{B}}\right) C^{ \pm}(Y \mid X)=0 .
$$

Indeed, since the time parameter $t=\frac{1}{M} X^{A B} T_{A B}$ is associated with any positive-definite $T_{A B}$, the sign in the exponential

$$
C(X)=C^{+}(X)+C^{-}(X), \quad C^{ \pm}(X)=\int d^{M} \xi c^{ \pm}(\xi) \exp \pm i \xi_{A} \xi_{B} X^{A B}
$$

is associated with the positive and negative frequencies. Hence, the unfolded equations for massless fields in $\mathcal{M}_{M}$ effectively quantize the model.

Another is the holographic duality between relativistic HS theory and nonrelativistic quantum mechanics. To this end, consider the reduction of Eq. (12.1) to the time arrow setting $X^{A B}=\delta^{A B} t$. The pullback of Eq. (12.1) to the time axis gives

$$
i \frac{\partial}{\partial t} C^{ \pm}(Y \mid t)= \pm \frac{\partial^{2}}{\partial Y^{A} \partial Y^{B}} \delta^{A B} C^{ \pm}(Y \mid t) .
$$

We observe that this equation has the form of the non-relativistic Schrodinger equation for a free particle in the space with coordinates $Y^{A}$. Indeed, its right-hand side acquires the form of Laplacian in the variables $Y^{A}$ while $C^{ \pm}$play a role of $\psi$ and $\bar{\psi}$.

By the general argument of the beginning of this section, the two systems are equivalent, i.e., the relativistic HS theory in the $X$-space is equivalent to the nonrelativistic theory in the twistor space. In particular, this equivalence manifests itself in the equivalence of their symmetry algebra. As demonstrated in [54, 55], the symmetry algebra of the Schrodinger equation is just the HS algebra of Section 6 .

The Schrodinger equation (12.2) has zero potential. An interesting question is what are dual HS theories for one or another nonzero potential. In the case of harmonic potential the answer is known [29]. The HS equations in $A d S$ and $d S$ space-times are dual to the quantummechanical models with the proper and upside down harmonic potentials, respectively. (Not surprisingly, the $d S$ geometry corresponds to the unstable quantum mechanics.)

Since the HS theory has a potential to unify gravity with quantum mechanics, one can speculate that it may be able to shed light on the both ingredients. Since full HS theory is nonlinear, its identification with quantum mechanics at the linearized level may suggest that, at ultrahigh energies, the HS theory may affect the fundamentals of quantum mechanics itself, making it nonlinear with the gravitationally small coupling constant! 


\section{To String Theory via Multiparticle Symmetry}

Properties of the HS theory are to large extent determined by the properties of the HS algebra. It has been long anticipated that the HS theory should be related somehow to String Theory. To materialize this idea it is most important to find a HS algebra rich enough to underly the full fledged String Theory. Recently it was conjectured [56] that such a symmetry can be associated with a multiparticle symmetry that acts on all multiparticle states of the HS theory.

Mathematically, this symmetry algebra can be defined as the Lie algebra associated with the universal enveloping algebra of the HS algebra of Section 6. It has a number of features that make it promising as a candidate for a string-like extension of the HS theory. In particular, it contains the original HS algebra as a subalgebra. Acting on all multiparticle states of HS theory it has enough room for mixed symmetry fields which appear in String Theory.

If this idea will indeed work, it will allow to interpret String Theory as a theory of bound states of the HS theory in striking analogy with the conjecture of [13].

\section{Summary and Conclusion}

The HS gauge theories contain gravity along with infinite towers of other fields with various spins including ordinary matter fields. An interesting feature of any HS model is that it always contains a scalar field associated with graviton, which carries no internal indices. It is tempting to speculate that this scalar may play a role in cosmology and, specifically, for inflation.

The HS theory contains non-minimal higher-derivative interactions that make it a kind of a nonlocal theory with unusual properties. In particular, many of the standard tools of GR based on Riemannian geometry may not be applicable to the HS theory as a consequence of the fact that the HS symmetry transforms a spin-two field to HS fields. In practice, this implies that in HS theories one has to be careful with the conventional interpretation of physical phenomena in terms of the metric tensor. In particular, this should be taken into account in the analysis of black hole physics in the framework of HS theory.

The HS gauge theories exist in any dimension [57]. However, the HS theories available so far are analogues of pure supergravity with no matter multiplets included. This makes it difficult to analyze the important issue of spontaneous breakdown of the HS symmetry which is necessary to introduce a mass scale analogous to the string tension. In fact, it can be argued that, to achieve a spontaneous breakdown of the HS symmetry, the string-like extension of the HS theory is needed. It was recently conjectured [56] that such an extension can be provided by a multiparticle theory to be identified with the quantum HS theory and String Theory.

Another exciting feature of the HS theory is that it exhibits a remarkable interplay between classical and quantum physics. This suggests that the further analysis may shed some light on both gravity and quantum mechanics at transplanckian energies which is the 
regime to be described by the HS theory.

HS theories not only have interesting holographic duals but also, being formulated in terms of unfolded dynamics approach, can shed light on the very origin of holographic duality. It can be argued [29] that the holographic duality links such models in space-times of different dimensions, that have equivalent form of their unfolded equations.

There are many important directions of the research of HS theories I had no chance to touch in these lectures.

One of the most interesting is the construction of exact solutions of HS equations. Most of exact solutions available so far, one way or another result from the solution of $3 d$ HS theory obtained in [39]. There are two main types of exact black-hole type solutions of the nonlinear HS equations available in the literature. The first one is represented by the flat connections associated with the BTZ-like black holes in the $3 d$ HS theory (see [58] and references therein; the interpretation of the usual BTZ black hole [59] as a solution of the HS equations was given in [60]). The second type includes black hole solutions in $A d S_{4}$ with the nonzero curvature tensor [61, 62]. Some other solutions were considered e.g. in [63, 64, 65]. Analysis of their properties in the context of the HS holographic duality and beyond is an important direction of the current research.

Among other activities we should mention analysis of the action principle in HS theory at the cubic level (see, e.g., [66, 67, 68, 69, 70, 71, 72]) and beyond [73, 74] as well as the further progress in understanding HS holography (see e.g., [75, 76, 77, 78, 79, 81, 80, 82, 83]) including holographic RG flows [84, 85, 86] and conformal correlators of HS currents [31, 87, 88, 89, 90, 91, 92].

Since in this short review it is hard even to list all important research directions in the HS theory, we refer the reader to other reviews [93, 94, 44, 95, 96, 30, 31, 58, 97] as well as to the contribution of Ricardo Troncoso to this workshop [98], where more detail and references on various aspects of the HS theory can be found.

\section{Acknowledgments}

The author is grateful to Olga Gelfond for useful comments on the manuscript and to the organizers of the 7th Aegean workshop on non-Einstein theories of gravity on Paros for the warm atmosphere and hospitality. This research was supported in part by RFBR Grant No 14-02-01172.

\section{References}

[1] D. Z. Freedman and A. Van Proeyen, Cambridge, UK: Cambridge Univ. Pr. (2012) $607 \mathrm{p}$

[2] A. H. Chamseddine and P. C. West, Nucl. Phys. B 129 (1977) 39.

[3] C. Fronsdal, Phys. Rev. D18 (1978) 3624. 
[4] S. Weinberg, Phys. Rev. 138 (1965) B988-B1002.

[5] S. R. Coleman and J. Mandula, Phys. Rev. 159 (1967) 1251-1256.

[6] C. Aragone and S. Deser, Phys. Lett. B86 (1979) 161.

[7] A. K. H. Bengtsson, I. Bengtsson, and L. Brink, Nucl. Phys. B227 (1983) 31.

[8] A. K. H. Bengtsson, I. Bengtsson, and L. Brink, Nucl. Phys. B227 (1983) 41.

[9] F. A. Berends, G. J. H. Burgers, and H. Van Dam, Z. Phys. C24 (1984) 247-254.

[10] F. A. Berends, G. J. H. Burgers, and H. van Dam, Nucl. Phys. B260 (1985) 295.

[11] E. S. Fradkin and M. A. Vasiliev, Phys. Lett. B189 (1987) 89-95.

[12] E. S. Fradkin and M. A. Vasiliev, Nucl. Phys. B291 (1987) 141.

[13] C. -M. Chang, S. Minwalla, T. Sharma and X. Yin, J. Phys. A 46 (2013) 214009 [arXiv:1207.4485 [hep-th]].

[14] J. M. Maldacena, Adv. Theor. Math. Phys. 2 (1998) 231 [Int. J. Theor. Phys. 38 (1999) 1113] [arXiv:hep-th/9711200].

[15] S. S. Gubser, I. R. Klebanov and A. M. Polyakov, Phys. Lett. B 428, 105 (1998) [arXiv:hep-th/9802109].

[16] E. Witten, Adv. Theor. Math. Phys. 2, 253 (1998) [arXiv:hep-th/9802150].

[17] S. E. Konstein, M. A. Vasiliev and V. N. Zaikin, JHEP 0012 (2000) 018 [hepth/0010239].

[18] B. Sundborg, Nucl. Phys. Proc. Suppl. 102 (2001) 113 [arXiv:hep-th/0103247].

[19] E. Witten, talk at the John Schwarz 60-th birthday symposium, http://theory.caltech.edu/jhs60/witten/1.html

[20] A. Mikhailov, arXiv:hep-th/0201019.

[21] E. Sezgin and P. Sundell, Nucl. Phys. B 644 (2002) 303 [Erratum-ibid. B 660 (2003) 403] [arXiv:hep-th/0205131].

[22] I. R. Klebanov and A. M. Polyakov, Phys. Lett. B 550 (2002) 213 [arXiv:hepth/0210114].

[23] S. Giombi and X. Yin, JHEP 1009 (2010) 115 [arXiv:0912.3462 [hep-th]].

[24] R. G. Leigh and A. C. Petkou, JHEP 0306 (2003) 011 [hep-th/0304217].

[25] E. Sezgin and P. Sundell, JHEP 0507 (2005) 044 [hep-th/0305040].

[26] M. Henneaux and S. -J. Rey, JHEP 1012 (2010) 007 [arXiv:1008.4579 [hep-th]].

[27] A. Campoleoni, S. Fredenhagen, S. Pfenninger and S. Theisen, JHEP 1011 (2010) 007 [arXiv:1008.4744 [hep-th]].

[28] M. R. Gaberdiel and R. Gopakumar, Phys. Rev. D 83 (2011) 066007 [arXiv:1011.2986 [hep-th]]. 
[29] M. A. Vasiliev, J. Phys. A 46 (2013) 214013 [arXiv:1203.5554 [hep-th]].

[30] M. R. Gaberdiel and R. Gopakumar, J. Phys. A 46 (2013) 214002 [arXiv:1207.6697 [hep-th]].

[31] S. Giombi and X. Yin, J. Phys. A 46 (2013) 214003 [arXiv:1208.4036 [hep-th]].

[32] O. V. Shaynkman and M. A. Vasiliev, Theor. Math. Phys. 128 (2001) 1155 [Teor. Mat. Fiz. 128 (2001) 378] [hep-th/0103208].

[33] M. G. Eastwood, Annals Math. 161 (2005) 1645 [hep-th/0206233].

[34] E. S. Fradkin and M. A. Vasiliev, Annals Phys. 177 (1987) 63.

[35] M. A. Vasiliev, Fortsch. Phys. 36 (1988) 33.

[36] M. A. Vasiliev, Annals Phys. 190 (1989) 59.

[37] M. A. Vasiliev, Phys. Lett. B 285 (1992) 225.

[38] S. E. Konshtein and M. A. Vasiliev, Nucl. Phys. B 312 (1989) 402.

[39] S. F. Prokushkin and M. A. Vasiliev, Nucl. Phys. B 545 (1999) 385 [hep-th/9806236].

[40] M. A. Vasiliev, Class. Quant. Grav. 8 (1991) 1387.

[41] M. A. Vasiliev, Int. J. Geom. Meth. Mod. Phys. 3 (2006) 37 [hep-th/0504090].

[42] M. A. Vasiliev, Nucl. Phys. B 793 (2008) 469 [arXiv:0707.1085 [hep-th]].

[43] T. Lada and J. Stasheff, Int. J. Theor. Phys. 32 (1993) 1087 [hep-th/9209099].

[44] X. Bekaert, S. Cnockaert, C. Iazeolla and M. A. Vasiliev, hep-th/0503128.

[45] R. J. Baston and M. G. Eastwood, Oxford, UK: Clarendon (1989) 213 p. (Oxford mathematical monographs)

[46] M. A. Vasiliev, Phys. Rev. D 66 (2002) 066006 [hep-th/0106149].

[47] O. A. Gelfond and M. A. Vasiliev, Theor. Math. Phys. 145 (2005) 1400 [Teor. Mat. Fiz. 145 (2005) 35] [hep-th/0304020].

[48] A. Jevicki, K. Jin and Q. Ye, J. Phys. A 46 (2013) 214005 [arXiv:1212.5215 [hep-th]].

[49] N. M. Nikolov, Y. S. Stanev and I. T. Todorov, J. Phys. A 35 (2002) 2985 [hepth/0110230].

[50] C. Fronsdal, "Massless Particles, Ortosymplectic Symmetry and Another Type of Kaluza-Klein Theory", Preprint UCLA/85/TEP/10, in Essays on Supersymmetry, Reidel, 1986 (Mathematical Physics Studies, v.8).

[51] M. A. Vasiliev, In *Olshanetsky, M. (ed.) et al.: Multiple facets of quantization and supersymmetry* 826-872 [hep-th/0111119].

[52] I. A. Bandos, J. Lukierski and D. P. Sorokin, Phys. Rev. D 61 (2000) 045002 [arXiv:hepth/9904109]. 
[53] I. Bandos, X. Bekaert, J. A. de Azcarraga, D. Sorokin and M. Tsulaia, JHEP 0505 (2005) 031 [hep-th/0501113].

[54] M. Valenzuela, arXiv:0912.0789 [hep-th].

[55] X. Bekaert, E. Meunier and S. Moroz, JHEP 1202 (2012) 113 [arXiv:1111.3656 [hepth]].

[56] M. A. Vasiliev, Class. Quant. Grav. 30 (2013) 104006 [arXiv:1212.6071 [hep-th]].

[57] M. A. Vasiliev, Phys. Lett. B 567 (2003) 139 [hep-th/0304049].

[58] M. Ammon, M. Gutperle, P. Kraus and E. Perlmutter, J. Phys. A 46 (2013) 214001

[59] M. Banados, C. Teitelboim and J. Zanelli, Phys. Rev. Lett. 69 (1992) 1849 [hepth/9204099].

[60] V. E. Didenko, A. S. Matveev and M. A. Vasiliev, Theor. Math. Phys. 153 (2007) 1487 [Teor. Mat. Fiz. 153 (2007) 158] [hep-th/0612161].

[61] V. E. Didenko and M. A. Vasiliev, Phys. Lett. B 682 (2009) 305 [Erratum-ibid. B 722 (2013) 389] [arXiv:0906.3898 [hep-th]].

[62] C. Iazeolla and P. Sundell, JHEP 1112 (2011) 084 [arXiv:1107.1217 [hep-th]].

[63] E. Sezgin and P. Sundell, Nucl. Phys. B 762 (2007) 1 [hep-th/0508158].

[64] E. Sezgin and P. Sundell, hep-th/0511296.

[65] C. Iazeolla, E. Sezgin and P. Sundell, Nucl. Phys. B 791 (2008) 231 [arXiv:0706.2983 [hep-th]].

[66] R. R. Metsaev, Nucl. Phys. B 759 (2006) 147 [hep-th/0512342].

[67] K. Alkalaev, JHEP 1103 (2011) 031 [arXiv:1011.6109 [hep-th]].

[68] M. A. Vasiliev, Nucl. Phys. B 862 (2012) 341 [arXiv:1108.5921 [hep-th]].

[69] I. L. Buchbinder, T. V. Snegirev and Y. .M. Zinoviev, Nucl. Phys. B 864 (2012) 694 [arXiv:1204.2341 [hep-th]].

[70] R. R. Metsaev, Phys. Lett. B 720 (2013) 237 [arXiv:1205.3131 [hep-th]].

[71] E. Joung, L. Lopez and M. Taronna, JHEP 1301 (2013) 168 [arXiv:1211.5912 [hep-th]].

[72] N. Boulanger, D. Ponomarev and E. D. Skvortsov, JHEP 1305 (2013) 008 [arXiv:1211.6979 [hep-th]].

[73] N. Boulanger and P. Sundell, J. Phys. A 44 (2011) 495402 [arXiv:1102.2219 [hep-th]].

[74] N. Boulanger, N. Colombo and P. Sundell, JHEP 1210 (2012) 043 [arXiv:1205.3339 [hep-th]].

[75] J. Maldacena and A. Zhiboedov, J. Phys. A 46 (2013) 214011 [arXiv:1112.1016 [hep-th]].

[76] J. Maldacena and A. Zhiboedov, Class. Quant. Grav. 30 (2013) 104003 [arXiv:1204.3882 [hep-th]]. 
[77] V. E. Didenko and E. D. Skvortsov, J. Phys. A 46 (2013) 214010 [arXiv:1207.6786 [hep-th]].

[78] H. Afshar, A. Bagchi, R. Fareghbal, D. Grumiller and J. Rosseel, Phys. Rev. Lett. 111 (2013) 121603 [arXiv:1307.4768 [hep-th]].

[79] S. Giombi and I. R. Klebanov, JHEP 1312 (2013) 068 [arXiv:1308.2337 [hep-th]].

[80] A. A. Tseytlin, Nucl. Phys. B 877 (2013) 598 [arXiv:1309.0785 [hep-th]].

[81] R. R. Metsaev, arXiv:1311.7350 [hep-th].

[82] S. Giombi, I. R. Klebanov and B. R. Safdi, arXiv:1401.0825 [hep-th].

[83] S. Giombi, I. R. Klebanov and A. A. Tseytlin, arXiv:1402.5396 [hep-th].

[84] M. R. Douglas, L. Mazzucato and S. S. Razamat, Phys. Rev. D 83 (2011) 071701 [arXiv:1011.4926 [hep-th]].

[85] I. Sachs, arXiv:1306.6654 [hep-th].

[86] R. G. Leigh, O. Parrikar and A. B. Weiss, arXiv:1402.1430 [hep-th].

[87] A. Zhiboedov, arXiv:1206.6370 [hep-th].

[88] N. Colombo and P. Sundell, arXiv:1208.3880 [hep-th].

[89] V. E. Didenko and E. D. Skvortsov, JHEP 1304 (2013) 158 [arXiv:1210.7963 [hep-th]].

[90] O. A. Gelfond and M. A. Vasiliev, Nucl. Phys. B 876 (2013) 871 [arXiv:1301.3123 [hep-th]].

[91] Y. S. Stanev, Nucl. Phys. B 876 (2013) 651 [arXiv:1307.5209 [hep-th]].

[92] I. Florakis, D. Sorokin and M. Tsulaia, arXiv:1401.1645 [hep-th].

[93] M. A. Vasiliev, Int. J. Mod. Phys. D 5 (1996) 763 [hep-th/9611024].

[94] M. A. Vasiliev, In *Shifman, M.A. (ed.): The many faces of the superworld* $533-610$ [hep-th/9910096].

[95] X. Bekaert, N. Boulanger and P. Sundell, Rev. Mod. Phys. 84 (2012) 987 [arXiv:1007.0435 [hep-th]].

[96] A. Sagnotti, J. Phys. A 46 (2013) 214006 [arXiv:1112.4285 [hep-th]].

[97] V. E. Didenko and E. D. Skvortsov, arXiv:1401.2975 [hep-th].

[98] A. Perez, D. Tempo and R. Troncoso, arXiv:1402.1465 [hep-th]. 